\title{
Metallothionein Prevents Age-Associated Cardiomyopathy via Inhibiting NF- $\kappa$ B Pathway Activation and Associated Nitrative Damage to 2-OGD
}

\author{
Weitao Cong, ${ }^{1, *}$ Chao Niu,, ${ }^{1, *}$ Lingchun Lv, ${ }^{2, *}$ Maowei Ni,, Dandan Ruan, ${ }^{4}$ Lisha Chi,, Yang Wang,, Qing Yu, \\ Kungao Zhan, ${ }^{6}$ Yuanhu Xuan, ${ }^{1}$ Yuehui Wang, ${ }^{7}$ Yi Tan, ${ }^{1,8}$ Tiemin Wei, ${ }^{2, \dagger}$ Lu Cai, ${ }^{7,8, \dagger}$ and Litai Jin ${ }^{1, \dagger}$
}

\section{Abstract}

Aims: Cardiac-specific metallothionein (MT) overexpression extends lifespan, but the mechanism underlying the effect of MT protection against age-associated cardiovascular diseases (CVD) remains elusive. To elucidate this, male wild-type and two lines of MT-transgenic (MT-TG) mice, MM and MT-1 (cardiac-specific overexpressing MT about 10- and 80-fold, respectively) at three representative ages (2-3, 9-10, and 18-20 months), were utilized. A stable human MT2A overexpressing cardiomyocytes (H9c2MT7) was also introduced.

Results: Histomorphology and echocardiographic analysis revealed that age-associated cardiac hypertrophy, remodeling, and dysfunction were ameliorated in MT-TG mice. Also, aging-accompanied NF- $\kappa$ B activation, characterized by increased nuclear p65 translocation, elevated DNA-binding activity, and upregulation of inflammatory cytokines, was largely attenuated by MT overexpression. Treatment of H9c2 cardiomyocytes with tumor necrosis factor- $\alpha$ (TNF- $\alpha)$, which mimicked an inflammatory environment, significantly increased NF- $\kappa$ B activity, and some age-related phenotypes appeared. The NF- $\kappa \mathrm{B}$ activation was further proved to be pivotal for both age-associated and TNF- $\alpha$-induced nitrative damage to cardiac 2-oxoglutarate dehydrogenase (2-OGD) by virtue of NF- $\kappa$ B p65 gene silencing. MT inhibited NF- $\kappa$ B activation and associated nitrative damage to cardiac 2-OGD in both old MT-TG hearts and TNF- $\alpha$-treated H9c2MT7 cardiomyocytes; these protective effects were abolished in H9c2MT7 cardiomyocytes by MT-specific gene silencing.

Innovation and Conclusion: Together, these findings indicate that the protective effects of MT against ageassociated CVD can be attributed mainly to its role in NF- $\kappa \mathrm{B}$ inhibition and resultant alleviation of nitrative damage to 2-OGD. Antioxid. Redox Signal. 25, 936-952.

Keywords: nitrative damage, metallothionein, 2-oxoglutarate dehydrogenase, inflammation, age-associated cardiovascular diseases

\section{Introduction}

A GING LEADS TO increased risks for various kinds of health complications, including cardiovascular diseases (CVD). Previous evidence demonstrated a drastic increase in the prevalence of atherosclerosis, hypertension, myocardial infarction, and stroke due to aging $(27,29)$.

Over the course of aging, cardiomyocytes become more susceptible to oxidative stress caused by the imbalance of redox system, resulting in the increasing rate of cardiomyocyte

\footnotetext{
${ }^{1}$ School of Pharmaceutical Science, Wenzhou Medical University, Wenzhou, P.R. China.

${ }^{2}$ The Fifth Affiliated Hospital of Wenzhou Medical University, Lishui, P.R. China.

${ }^{3}$ Zhejiang Cancer Hospital, Hangzhou, P.R. China.

${ }^{4}$ The Health Examination Center, the 117th Hospital of Chinese People's Liberation Army, Hangzhou, P.R. China.

${ }^{5}$ Department of Histology and Embryology, Institute of Neuroscience, Wenzhou Medical University, Wenzhou, P.R. China.

${ }^{6}$ The Second Affiliated Hospital of Wenzhou Medical University, Wenzhou, P.R. China.

${ }^{7}$ The First Hospital of Jilin University, Changchun, P.R. China.

${ }^{8}$ Department of Pediatrics, Kosair Children's Hospital Research Institute, University of Louisville, Louisville, Kentucky.

*Co-first authors.

${ }^{\dagger}$ Co-senior authors.
} 


\section{Innovation}

We observed for the first time that 2-oxoglutarate dehydrogenase complex (2-OGDHC) nitration and its subsequent dysfunction are involved in aging, providing a link between inflamm-aging and age-associated cardiovascular diseases (CVD). Moreover, we showed that the functional importance of metallothionein (MT) is not limited to its role as a defender against oxidative stress. Instead, its novel role in inhibiting NF- $\kappa \mathrm{B}$ activation and the resultant nitrative damage to 2-OGD ensure that cardiac cells have an adequate energy supply. Thus, MT plays a crucial role in preventing aging-associated CVD.

death (8). When cardiomyocyte necrosis occurs, a release of cellular components is accompanied, which affects the viability of cells in the vicinity. This process also promotes the development of proinflammatory environments (32), which could further enhance oxidative stress and activate the reactive oxygen species (ROS)-dependent NF- $\kappa \mathrm{B}$ pathway (15).

Of note, the highly oxidative inflammatory environment induces oxidative modifications to kinds of biomacromolecules (7), of which the protein oxidation is especially hazardous as the consequent damage and conformational alterations in structures can make target proteins inactive, resulting in functional abnormalities $(2,7)$. Consistent with this view, a number of important enzymes that are involved in glycolysis, gluconeogenesis, and the citrate cycle have been demonstrated to be modified during aging $(3,6)$. These results suggest a potential link between protein oxidation and the disturbance of cellular energy metabolism.

The aging process is linked with a subclinical systemic inflammatory response, referred to as "inflamm-aging," which was first proposed and explained by Franceschi $e t$ al. (20). Inflammatory cytokines such as tumor necrosis factor- $\alpha$ $(\mathrm{TNF}-\alpha)$ induce the production of nitric oxide (NO) by activating inducible nitric oxide synthase (iNOS) (36). The dual activation of superoxide- and NO-generating systems renders the intracellular environment favorable for the formation of peroxynitrite $\left(\mathrm{ONOO}^{-}\right)$and results in the nitration of tyrosine-containing proteins (42), and 3-nitrotyrosine (3-NT) is, thus, formed. More importantly, studies in animals and cultured cells demonstrated that 3-NT is a crucial pathogenic agent of CVD during oxidative stress (18).

Metallothionein (MT) is a family of metal-binding proteins harboring high numbers of cysteine residues, which makes it an important component for defending against oxidative stress. Downregulation of oxidative stress-mediated apoptosis has been demonstrated (17). Moreover, MT prevents the inflammatory cascades from initiating after tissue injury (24), and it also modulates metabolism (46). Over the past few years, extensive studies have drawn much attention to the role of MT in modulating lifespan, highlighting it as an attractive target for aging research (44). To date, however, the molecular mechanism underlying the protective effect of MT on age-associated CVD remains unclear.

This study aimed at investigating how the agingaccompanied inflammatory response was mediated by MT overexpression, and its related effects in old mice hearts. Additionally, MT-mediated alleviation of both age-associated and TNF- $\alpha$-induced nitrative damage to certain proteins was also analyzed for further elaborating the protective role of MT in age-associated CVD.

\section{Results}

\section{Cardiac function and remodeling were partially} preserved in old MT-transgenic mice

Hematoxylin-eosin and Sirius red staining revealed that aging is accompanied by myocardial structure disturbance, myofibrillar discontinuation, and enhanced fibrosis (Fig. 1A). The extent of myocardial remolding clearly increased with aging in the hearts of wild-type (WT) mice, but to a significantly lesser extent in MT-transgenic (TG) mice.

Echocardiographic assessment of in vivo left ventricular (LV) function was performed by using the two lines of MT-TG mice at ages of 2-3 and 18-20 months (hereafter, "young" and "old," respectively). LV end-systolic dimensions (LVESD), LV end-diastolic dimensions (LVEDD), fractional shortening (FS\%), and ejection fraction (EF\%) were similar between the young MT-TG and WT mice (Fig. 1B).

In old WT mice, the LV was significantly dilated (higher LV long-axis diameter at end-diastole and end-systole stages). Mild LV systolic dysfunction (reduced FS\% and EF\%) was also observed in old WT mice. By contrast, these phenotypes were alleviated in the same-age MT-TG mice. Similarly, a remarkable increase in heart weight normalized to body weight (HW/BW) was observed in old WT mice, but not in old MTTG mice (Fig. 1B and Supplementary Table S1; Supplementary Data are available online at www.liebertpub.com/ars).

\section{Senescence-associated phenotypes were partially alleviated in old MT-TG mice}

Cellular senescence, which is considered the hallmark of aging, is characterized by phenotypes, including elevated senescence-associated beta-galactosidase (SA- $\beta$-gal) activity and increased expression of cell-cycle inhibitors (34). As shown in Figure 1C, SA- $\beta$-gal activity was markedly elevated in old WT mice, as reflected by the extended SA- $\beta$-gal-positive area, but it was significantly lower in old MT-TG mice.

In many organs of rodents, expression of INK4/ARF family proteins increases with age (26). We confirmed that levels of $\mathrm{p} 16^{\mathrm{INK} 4 \mathrm{a}}$ and $\mathrm{p} 19^{\mathrm{ARF}}$ increased with age in WT mice, but they remained low in old MT-TG mice (Fig. 1D). These results demonstrated that cellular senescence, to some extent, could be prevented by MT overexpression.

\section{MT prevents cardiac NF- $\kappa B$ activation and the subsequent inflammatory response in old mice hearts}

Inflammation is a crucial etiological factor in the progression of several age-associated diseases, including ageassociated CVD. Accordingly, we analyzed inflammatory cell infiltration during the aging process. As shown in Figure 2A, the number of myeloperoxidase (MPO)-peroxidase+ neutrophils increased significantly in old WT hearts. Fewer MPO+ cells were present in old MT-TG hearts, indicating that MT overexpression effectively attenuated this increase.

Next, we compared the levels of proinflammatory cytokines in myocardial tissue (Fig. 2B and Supplementary Fig. S1A) and plasma (Supplementary Fig. S1B) of young and old mice. The protein levels of TNF- $\alpha$, interleukin- $1 \beta$ (IL- $1 \beta$ ), and IL-6 were elevated in both myocardial tissue and 

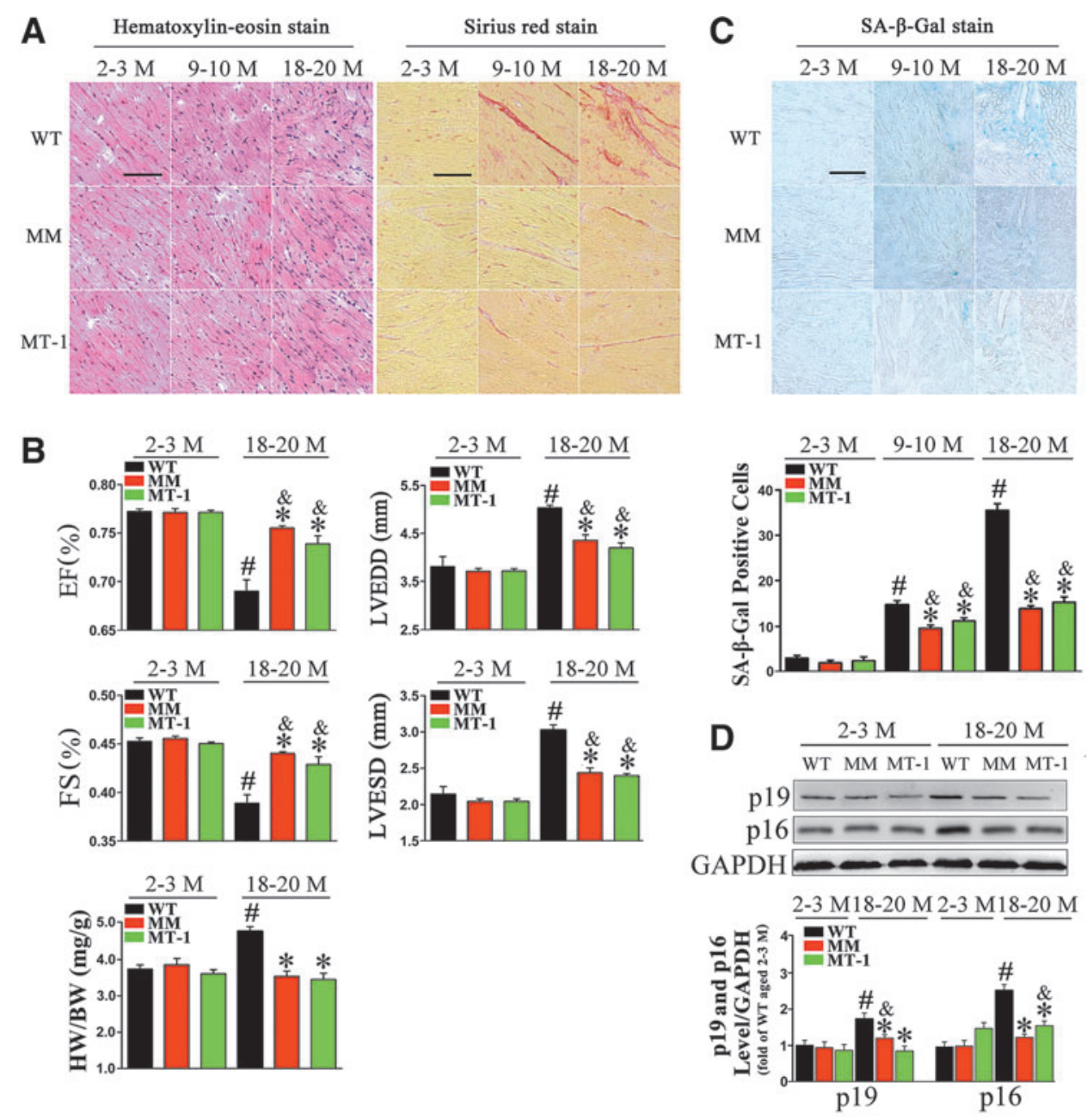

FIG. 1. Overexpression of MT protects cardiac function, remodeling and alleviates age-associated phenotype in old mice hearts. (A) Heart tissue sections from WT and MT-TG (MM, MT-1) mice at three representative age stages (2-3, 9-10, and 18-20 months) were used for hematoxylin-eosin staining and Sirius red staining, respectively; $n=6$ in each group. Scale bars $=100 \mu \mathrm{m}$. (B) Transthoracic echocardiography was performed in WT and MT-TG (MM, MT-1) mice at mean ages of 2-3 and 18-20 months. LVESD, LVEDD, FS\%, EF\%, and HW/BW were measured; $n=9$ in each group. (C) Frozen heart tissue sections from WT and MT-TG (MM, MT-1) mice at three representative stages (2-3, 9-10, and 18-20 months) were analyzed for SA- $\beta$-gal staining. The histogram (lower panel) is the quantitative analysis of the SA- $\beta$-gal-positive area in at least six separate fields; $n=6$ for each group. Scale bars $=100 \mu \mathrm{m}$. (D) The protein levels of cell-cycle inhibitors (senescence markers) p19 and p16 in WT and MT-TG (MM, MT-1) at mean ages of 2-3 and 18-20 months were examined via immunoblotting. The histogram (lower panel) is the quantitative analysis of the corresponding immunoblots, and the data are expressed as fold over WT mice at $2-3$ months; $n=6$ for each group. Data are presented as mean \pm SD. ${ }^{\#} p<0.05$ versus WT mice at $2-3$ months old; ${ }^{*} p<0.05$ versus WT mice in the same age stage; ${ }^{*} p<0.05$ versus the same genotype of MT-TG mice at $2-3$ months old. EF, ejection fraction; FS\%, percent fractional shortening; HW/BW, heart weight normalized to body weight; LVEDD, left ventricular end-diastolic dimensions; LVESD, left ventricular end-systolic dimensions; MT-TG, metallothionein-transgenic; SA- $\beta$-gal, senescence-associated beta-galactosidase; SD, standard deviation; WT, wild type.

plasma of old mice. However, cardiac-specific MT overexpression decreased the levels of proinflammatory cytokines in myocardial tissue from old hearts without altering the corresponding plasma levels.

In light of the effect on cytokine production, we explored NF- $\kappa$ B activity. Nuclear extracts from young and old WT and MT-TG hearts were subjected to electrophoretic mobility shift assay (EMSA) for NF- $\kappa$ B DNA-binding activity assessment (Fig. 2C). DNA-binding activity of NF- $\kappa$ B was elevated in old WT hearts, but it remained low in old MT-TG hearts. To investigate the underlying molecular mechanism, we performed immunoblots of p65 (Fig. 2D). In the WT, the nuclear translocation of p65 was obviously higher in old hearts than in young hearts. Consistently, MT suppressed age-associated nuclear translocation of p65. Furthermore, MT inhibited $\mathrm{I} \kappa \mathrm{B}$ phosphorylation and its subsequent degradation, which is the last step for NF- $\kappa \mathrm{B}$ activation in the cytoplasm (40) (Fig. 2E).

To further explore the inhibitory effects of MT during the inflammatory process, we investigated the effect of MT on the cardiac response to lipopolysaccharide (LPS) (Supplementary Fig. S2). Results showed that MT overexpression significantly suppressed LPS-induced nuclear translocation of p65, along with an attenuated DNA-binding activity of NF- $\kappa$ B in MT-TG 
A
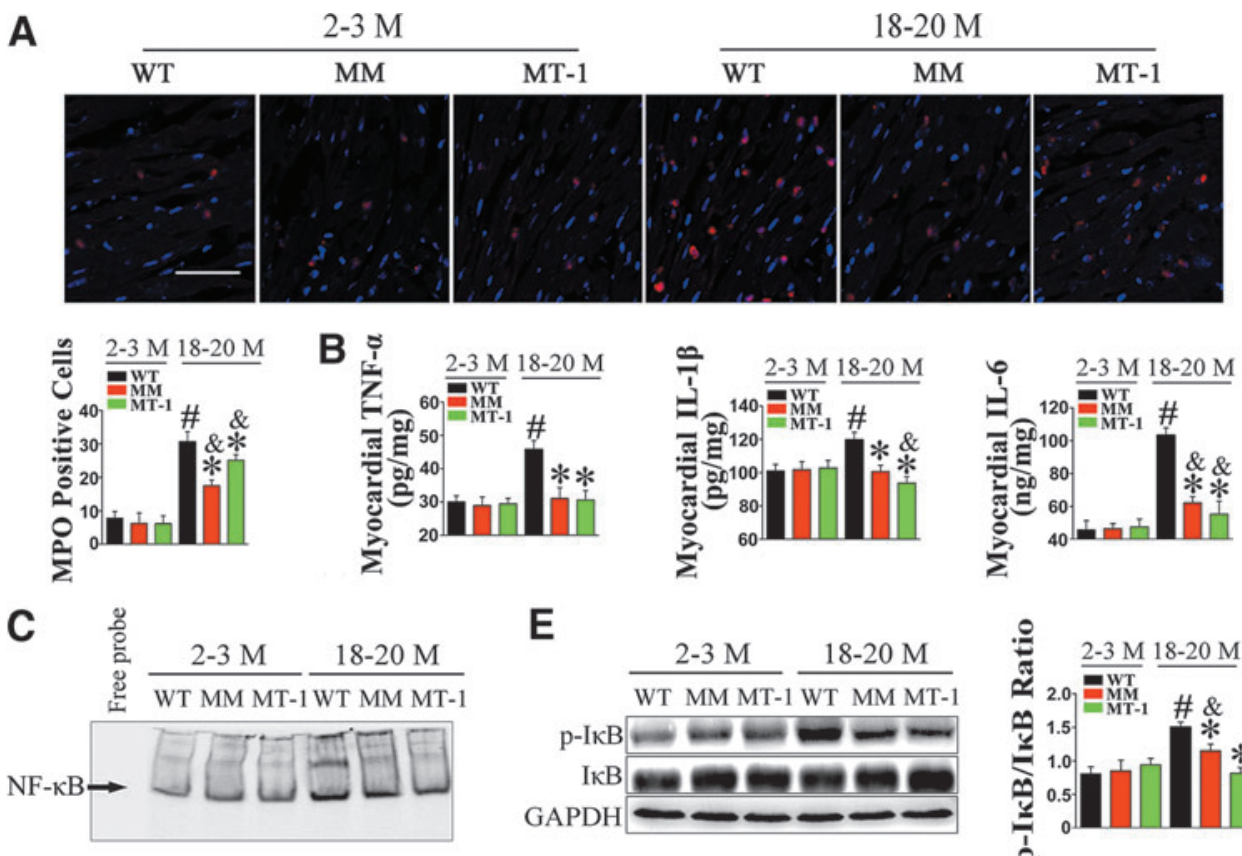

E
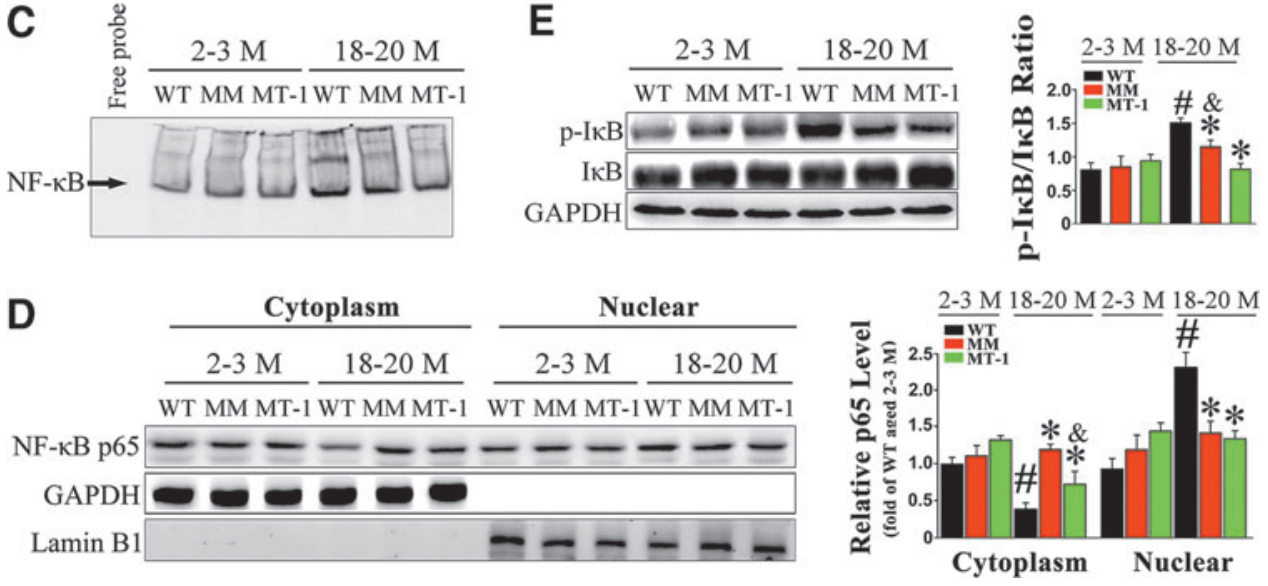

FIG. 2. MT prevents cardiac NF- $\kappa \mathrm{B}$ activation and the subsequent inflammatory response in old mice hearts. (A) Representative images in frozen heart tissue sections showing infiltration of the myocardium with neutrophils indicated by MPO-peroxidase immunofluorescence staining in WT and MT-TG (MM, MT-1) at mean ages of 2-3 and 18-20 months. The histogram (lower panel) is the quantitative analysis of MPO+ cells in at least six separate fields; $n=6$ for each group. Scale bars $=50 \mu \mathrm{m}$. (B) Levels of TNF- $\alpha$, IL-1 $\beta$, and IL-6 in the myocardium of WT and MT-TG (MM, MT-1) at mean ages of 2-3 and 18-20 months were measured by ELISA. (C) Nuclear extracts made from the hearts of WT and MT-TG (MM, MT-1) at mean ages of 2-3 and 18-20 months were subjected to EMSA to measure NF- $\kappa$ B DNA-binding activity, and a representative EMSA gel is presented; $n=6$ for each group. (D) Nuclear and cytosolic extracts were isolated, and the nuclear translocation of p65 was analyzed by immunoblotting. The histogram (right panel) is the quantitative analysis of the corresponding immunoblots, and the data are expressed as fold over WT mice at 2-3 months; $n=6$ for each group. (E) Immunoblots of heart homogenates from WT and MT-TG (MM, MT-1) mice at mean ages of 2-3 and 18-20 months were probed for phosphor-I $\kappa$ B $\alpha$ and total $\mathrm{I} \kappa \mathrm{B} \alpha$ expression. The histogram (right panel) is the quantitative result of $\mathrm{pI} \kappa \mathrm{B} \alpha / \mathrm{I} \kappa \mathrm{B} \alpha$ ratio; $n=6$ for each group. Data are presented as mean \pm SD. ${ }^{\#} p<0.05$ versus WT mice at $2-3$ months old; ${ }^{*} p<0.05$ versus WT mice in the same age stage; ${ }^{\&} p<0.05$ versus the same genotype of MT-TG mice at $2-3$ months old. ELISA, enzyme-linked immunosorbent assay; EMSA, electrophoretic mobility shift assay; IL, interleukin; MPO, myeloperoxidase; TNF- $\alpha$, tumor necrosis factor- $\alpha$.

hearts (Supplementary Fig. S2A, B). The elevation of cardiac transcription levels of TNF- $\alpha$, IL-1 $\beta$, and IL-6 after LPS injection was effectively alleviated in MT-TG hearts (Supplementary Fig. S2C).

Furthermore, LPS-induced cardiac apoptosis was also suppressed by the overexpression of MT (Supplementary Fig. S2D). Therefore, these results confirmed the role of MT in protecting against the activation of NF- $\kappa \mathrm{B}$.

\section{MT prevents cardiac apoptosis in old mice}

Terminal deoxynucleotidyl transferase dUTP-mediated nick-end labeling (TUNEL) analysis revealed that the cardiac apoptosis rate was higher in old WT hearts than in young WT hearts. This effect was significantly alleviated in old MT-TG hearts (Fig. 3A).
We further tested the protective effect of MT on agingassociated cardiac apoptosis by examining caspase- 3 activity, cleaved caspase- 3 expression, and $\mathrm{Bax} / \mathrm{Bcl}-2$ ratio; consistent results were obtained in all cases (Fig. 3B-D). In addition, MT promoted cellular survival in old hearts, as demonstrated by the elevated ratio of phospho-AKT to total AKT (Fig. 3E).

\section{3-NT modification of 2-oxoglutarate dehydrogenase is involved in cardiac aging, whereas MT inhibits age-associated nitrative damage, thereby preserving enzyme activity and energy equilibrium in the old heart}

Oxidative stress plays a key role in inflamm-aging and associated diseases (23). We observed more intense fluorescence-labeled dihydroethidium (DHE) staining in the 
A
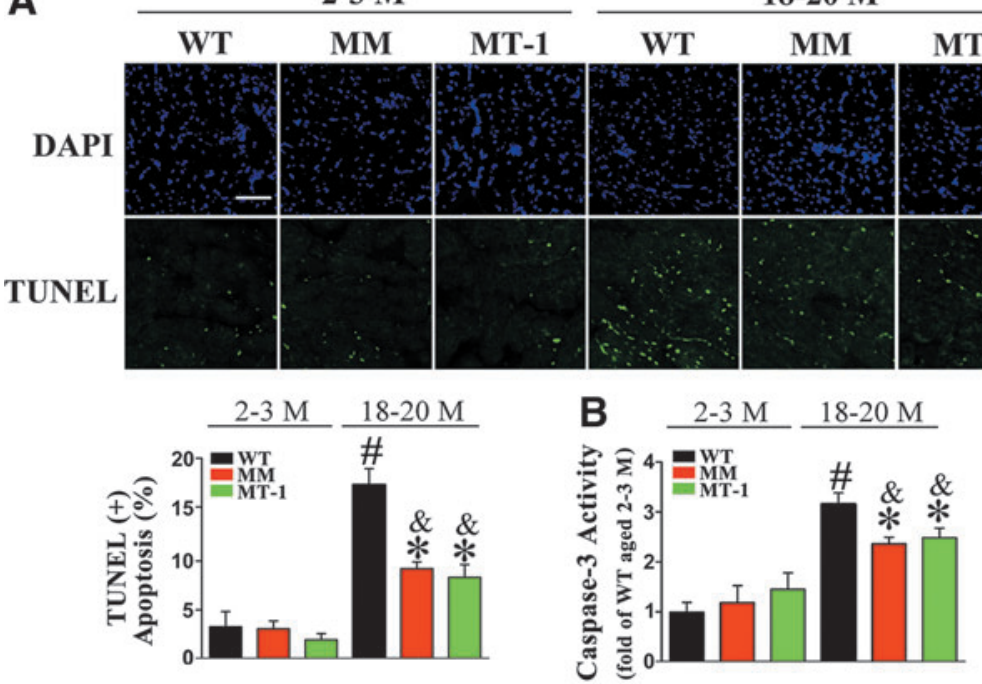

C

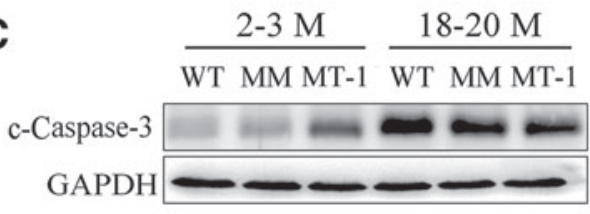

D

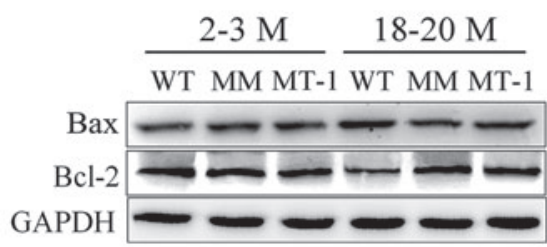

E

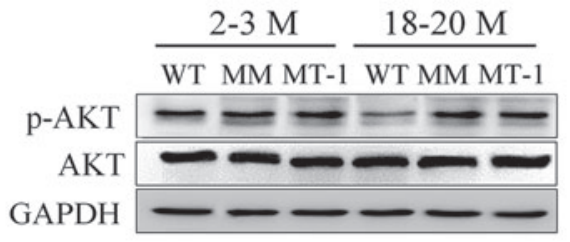

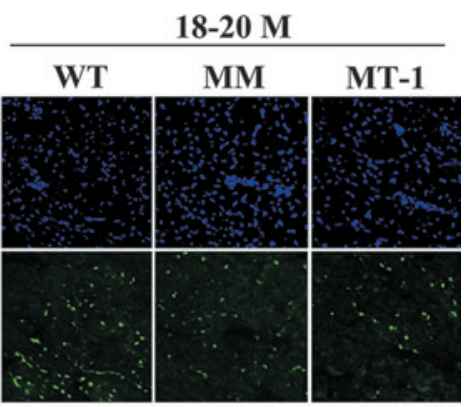
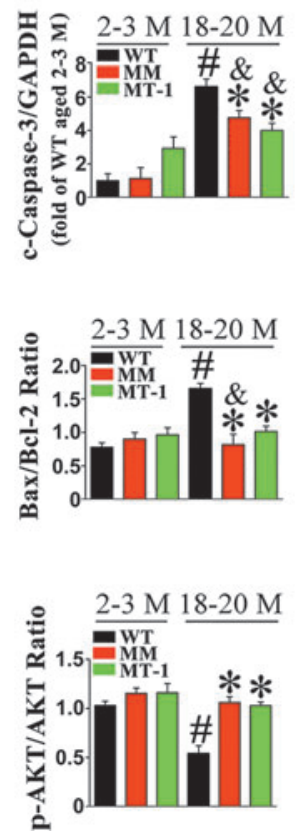

FIG. 3. MT prevents cardiac apoptosis in old mice. (A) DAPI (top) staining and TUNEL assay (bottom) of the frozen heart tissue sections from WT and MT-TG (MM, MT-1) mice at mean ages of 2-3 and 18-20 months. The histogram (lower panel) is the quantitative analysis of TUNEL+ cells in at least six separate fields; $n=6$ for each group. Scale bars $=50 \mu \mathrm{m}$. (B) Caspase-3 activity assay of the heart homogenates from WT and MT-TG (MM, MT-1) mice at mean ages of 2-3 and 18-20 months, and the data are expressed as fold over WT mice at 2-3 months; $n=6$ for each group. (C-E) Immunoblotting assays of cleaved-caspase-3 expression, Bax/Bcl-2, and phosphor-AKT/ total AKT ratio in the hearts from WT and MT-TG (MM, MT-1) mice at mean ages of 2-3 and 18-20 months. The histograms (right panel) are the quantitative analysis of each immunoblots, and the data of cleavedcaspase-3 are expressed as fold over WT mice at 2-3 months; $n=6$ for each group. Data are presented as mean \pm SD. $\# p<0.05$ versus WT mice at $2-3$ months old; $* p<0.05$ versus WT mice in the same age stage; ${ }^{*} p<0.05$ versus the same genotype of MT-TG mice at 2-3 months old. DAPI, 4', 6-diamidino-2-phenylindole; TUNEL, terminal deoxynucleotidyl transferase dUTPmediated nick-end labeling. hearts of old WT mice in comparison with young animals, suggesting elevated accumulation of superoxide with aging. By contrast, old MT-TG mice had lower DHE fluorescent intensity (Fig. 4A).

In addition, the level of iNOS in heart homogenates increased dramatically (Fig. 4B), concomitant with elevated nitrite production, during the aging process (Supplementary Fig. S3A). Hyperproduction of NO due to induction of iNOS leads to the formation of $\mathrm{ONOO}^{-}$, which can react with particular residues in proteins, thereby affecting their biological function (19). Therefore, we assessed the possible involvement of nitrative damage in age-associated CVD and the effect of MT on 3-NT formation in WT and MT-TG mice hearts with mean ages of 2-3, 9-10 (hereafter, "middleaged"), and 18-20 months.

In WT mice, quantitative analysis by immunoblotting revealed that 3-NT accumulation was much higher in old mice than in young mice. 3-NT was predominantly present in proteins of $\sim 115 \mathrm{kDa}$ (Fig. 4C), where 2-oxoglutarate dehydrogenase (2-OGD) are located (Supplementary Table S2). In contrast to WT mice, old MT-TG mice exhibited less 3-NT modification in proteins of $\sim 115 \mathrm{kDa}$ (Fig. 4C). In addition, we also identified other less nitrated proteins in old hearts (Supplementary Fig. S4 and Supplementary Table S3).

To further confirm the nitrative damage of 2-OGD, we examined the protein level of 2-OGD in young, middle-aged, and old MT-TG and WT mice hearts. We observed no obvious up/downregulation of 2-OGD expression level in any age group (Fig. 4D). Heart tissue samples were then immunoprecipitated with anti-2-OGD antibody and blotted with anti-3-NT antibody to examine the expression level of 3-NTmodified 2-OGD (Fig. 4D). The results confirmed an agedependent increase in the modification of 3-NT to 2-OGD in WT mice, which was markedly suppressed in MT-TG mice. The age-associated nitration of 2-OGD was not detected in 
A

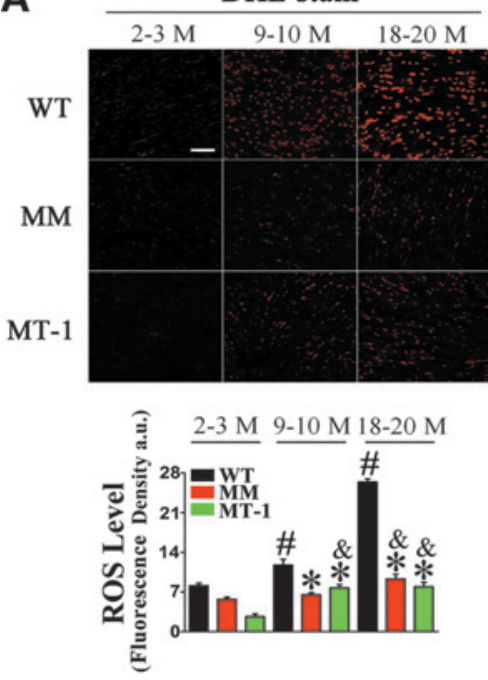

C IB: 3-NT $\quad 2-3 \mathrm{M} \quad$ 9-10 M $18-20 \mathrm{M}$ $\overline{\text { WT MM MT-1 WT MM MT-1 WT MM MT- }}$

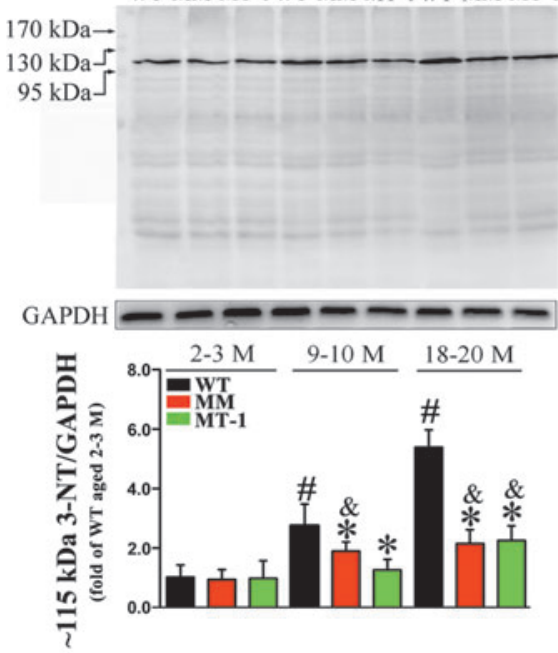

B

B $2-3 \mathrm{M} \frac{9-10 \mathrm{M}}{18-20 \mathrm{M}}$ $\overline{\text { WT MM MT-1 WT MM MT-1 WT MM MT-1 }}$ iNOS GAPDH
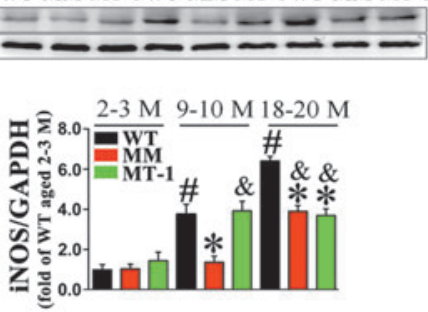

D
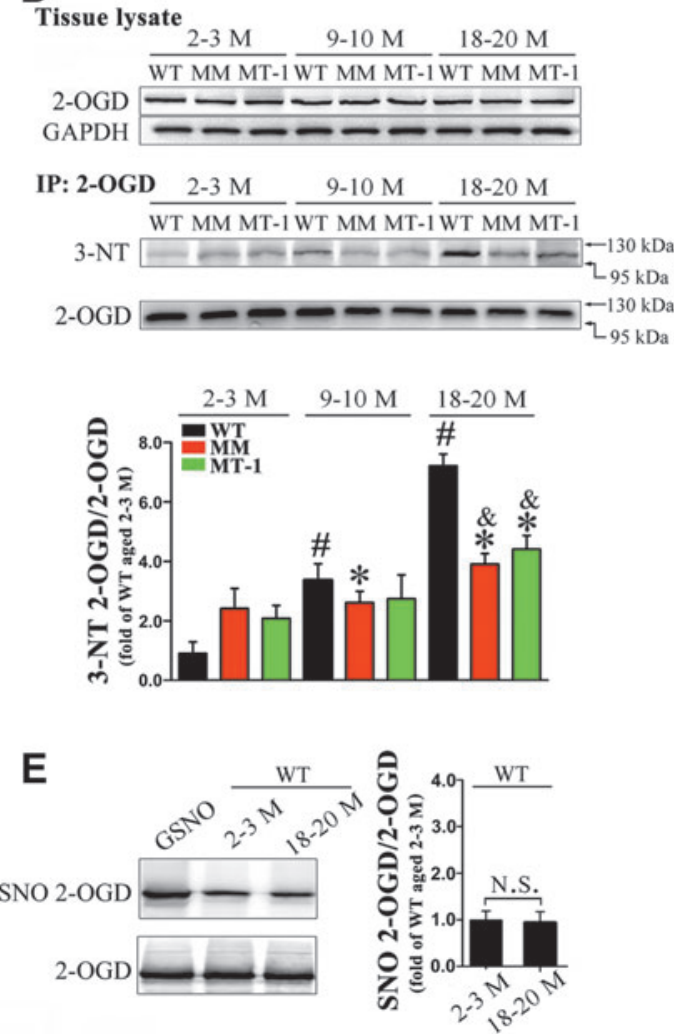

FIG. 4. 3-NT modification of 2-OGD is involved in the process of heart aging, whereas MT could inhibit ageassociated 3-NT accumulation. (A) DHE staining was performed in the frozen heart tissue sections from WT and MT-TG (MM, MT-1) mice at three representative age stages (2-3, 9-10, and 18-20 months). The histogram (lower panel) is the quantitative analysis of the fluorescence density in at least six separate fields, and the fluorescence density is represented as A.U.; $n=6$ for each group. Scale bars $=50 \mu \mathrm{m}$. (B) Immunoblots of heart homogenates from WT and MT-TG (MM, MT-1) mice at three representative stages (2-3, 9-10, and 18-20 months) to examine iNOS levels; $n=6$ for each group. (C) Protein nitration detected by immunoblotting using heart tissues from WT and MT-TG (MM, MT-1) mice at mean ages of 2-3, 910, and 18-20 months, and 3-NT was predominantly present in proteins with molecular weights of $\sim 115 \mathrm{kDa}$. The histograms (lower panel) in (B) and (C) are the quantitative analysis of the corresponding immunoblots, and the data are expressed as fold over WT mice at 2-3 months; $n=6$ for each group. (D) Immunoblots of heart homogenates from WT and MT-TG (MM, MT-1) mice at three representative stages (2-3, 9-10, and 18-20 months) to examine total 2-OGD levels. Then, the heart homogenates were subjected to immunoprecipitation with 2-OGD antibody, followed by immunoblotting with 3-NT and 2-OGD-specific antibody. The histogram (lower panel) is the immunoblotting quantitative analysis of 3-NT 2-OGD/2-OGD, and the data are expressed as fold over WT mice at 2-3 months; $n=6$ for each group. (E) The biotin-switch method was performed to detect S-nitrosation in 2-OGD (SNO-2-OGD) in the hearts of WT mice at 2-3 and 18-20 months. The heart extracts from WT mice at 2-3 months old were incubated with $40 \mu M$ S-nitroso-glutathione (GSNO) for 30 min as a positive control. The histograms (right panel) are the quantitative analysis of the corresponding immunoblots, and the data are expressed as fold over WT mice at $2-3$ months; $n=6$ for each group. Data are presented as mean \pm SD. ${ }^{\#} p<0.05$ versus WT mice at $2-3$ months old; ${ }^{*} p<0.05$ versus WT mice in the same age stage; ${ }^{*} p<0.05$ versus the same genotype of MTTG mice at 2-3 months old; N.S., non-significant. 2-OGD, 2-oxoglutarate dehydrogenase; 3-NT, 3-nitrotyrosine; A.U., arbitrary units; DHE, dihydroethidium; iNOS, inducible nitric oxide synthase. 
other tissues (kidney, brain, and liver) from old WT mice, suggesting a unique role for 2-OGD nitration in old hearts (Supplementary Fig. S5A-D).

Considering that the thiol-containing cysteine in redoxsensitive proteins is particularly sensitive to alterations in the redox environment $(9,16)$, the possible role of cysteine oxidation in 2-OGD during cardiac aging was assayed. Accordingly, we used the biotin-switch method to examine Snitrosation, a reversible NO-induced modification, on the cysteine in 2-OGD (Fig. 4E). We observed no apparent difference of S-nitrosation on 2-OGD between young and old WT hearts. Furthermore, the irreversible cysteine oxidation in 2-OGD (sulfinic and sulfonic acid formation) was also assessed by MS analysis, but only tyrosine nitration in 2OGD was detected in old WT hearts (Supplementary Table S2).

To determine whether the elevated rate of nitration adduction affected the function of 2-oxoglutarate dehydrogenase complex (2-OGDHC), we monitored 2-OGDHC activity in isolated cardiac mitochondrial extracts. As shown in Supplementary Figure S6A, 2-OGDHC activity declined with aging. In WT mice, enzyme activity did not differ significantly between middle-aged and young animals; whereas in old mice, cardiac 2-OGDHC activity was reduced by $\sim 30 \%$.

Moreover, kinetics analysis of 2-OGDHC revealed that $K_{\mathrm{m}}$ for substrate a-ketoglutarate and $V_{\max }$ varied among age groups (Supplementary Fig. S7 and Supplementary Table S4). In contrast with that in young mice, the $K_{\mathrm{m}}$ in middle-aged and old mice was elevated by $\sim 18 \%$ and $\sim 34 \%$, and $V_{\max }$ was reduced by $\sim 18 \%$ and $\sim 26 \%$, respectively. Together, these observations indicate a reduced affinity for the substrate and slower catalysis in older animals. On the other hand, in MT-TG mice, enzyme kinetic characteristics were well preserved with advancing age. These results suggest a possible link between 2-OGD nitration and 2-OGDHC dysfunction.

In view of the fact that 2-OGDHC is a rate-limiting enzyme of the tricarboxylic acid (TCA) cycle, which is functionally connected to NADH-linked respiration and energy production, we examined the total ATP level in hearts and $\mathrm{NADH}$ content in isolated mitochondria (Supplementary Fig. S6B, C). In WT hearts, we observed an obvious decrease in NADH production over the course of aging, especially in old mice. In addition, we observed a similar trend in total ATP content, as determined by luciferin/luciferase assay. By contrast, heart-specific MT overexpression could partially improve these age-dependent conditions.

To further monitor the influence of 2-OGD nitration on overall energy metabolism, we used ion-pairing LC-MS to analyze the energy charge in each age group. The results revealed that energy charge declined mildly $(\sim 6 \%)$ in middle-aged WT mice, whereas a $\sim 16 \%$ decline in old WT mice was noted with significant difference. In MT-TG mice, energy charge was well preserved, even in old mice (Supplementary Fig. S6D).

\section{$N F-\kappa B$ inhibition acts as a major role in the attenuation of nitrative damage to 2-OGD}

Our in vivo results indicated that the protective effects of MT against aging-associated CVD might be attributed to its role in inhibiting the $\mathrm{NF}-\kappa \mathrm{B}$ pathway. However, we lacked direct proof of the link between NF- $\kappa \mathrm{B}$ activity and subsequent nitrative damage during aging.

To obtain this, we directly injected lentiviral plasmids containing sh-p65 (LRV-U6-p65-CMV-EGFP) and a scrambled sequence (LRV-U6-scramble-CMV-EGFP) into the myocardium of 15-month-old WT mice over a period of 12 weeks, and the transfection was confirmed by the presence of EGFP in the myocardium (Fig. 5A). L-sh-p65 injection dramatically suppressed NF- $\kappa \mathrm{B}$ p65 expression in WT hearts (Fig. 5B). As expected, iNOS protein was downregulated after L-sh-p65 treatment (Fig. 5C). L-sh-p65-treated WT mice also exhibited significant attenuation of aging-induced nitrative damage to 2-OGD in comparison with the scrambletreated or non-treated group (Fig. 5D). Meanwhile, 2OGDHC activity was efficiently restored in WT hearts due to p65 knockdown by L-sh-p65 (Fig. 5E).

\section{$M T$ inhibits $T N F-\alpha$-induced $N F-\kappa B$ activation and aging phenotypes in vitro}

To further elucidate the relationship between inflammation and aging, we administered exogenous TNF- $\alpha$ to H9c2 and H9c2MT7 cardiomyocytes for $24 \mathrm{~h}$ to mimic an inflammatory environment. To measure ROS production in the cytosol, we transfected both cell lines with the cytosolic-localized redox-sensitive GFP mutant (Cyto-RoGFP) (Fig. 6A). In $\mathrm{H} 9 \mathrm{c} 2$ cardiomyocytes, $\mathrm{TNF}-\alpha$ treatment significantly increased the fluorescence ratio $(400 \mathrm{~nm} / 484 \mathrm{~nm})$, suggesting that the ROS level in the cytosol was elevated. In addition, NF- $\kappa$ B DNA-binding activity, nuclear p65 translocation, and $\mathrm{I} \kappa \mathrm{B}$ degradation were also increased by TNF- $\alpha$ treatment in $\mathrm{H} 9 \mathrm{c} 2$ cardiomyocytes (Fig. 6B-D), resulting in higher levels of proinflammatory cytokines in both lysate and culture medium (Supplementary Fig. S8A, B).

By contrast, when $\mathrm{H} 9 \mathrm{c} 2$ cardiomyocytes were treated for $24 \mathrm{~h}$ with TNF- $\alpha$ in the presence of $100 \mu \mathrm{M}$ urate, an $\mathrm{ONOO}^{-}$ scavenger, or $100 \mu M \mathrm{Mn}(\mathrm{III})$ tetrakis 1-methyl 4-pyridyl porphyrin pentachloride (MnTMPyP), an SOD mimic, the cytosolic ROS level was markedly reduced (Fig. 6A). Importantly, NF- $\kappa$ B DNA-binding activity, nuclear p65 translocation, and $\mathrm{I} \kappa \mathrm{B}$ degradation were subsequently inhibited (Fig. 6B-D), suggesting that NF- $\kappa \mathrm{B}$ activation was ROS dependent.

In addition, some age-related phenotypes, such as an increase in SA- $\beta$-gal-positive cardiomyocytes (Fig. 6E) and upregulation of the senescence markers $\mathrm{p} 16^{\mathrm{INK} 4 \mathrm{a}}$ and $\mathrm{p} 19^{\mathrm{ARF}}$ (Fig. 6F), were observed in TNF- $\alpha$-treated H9c2 cardiomyocytes. When the cells were pretreated with Bay-117082, an $\mathrm{NF}-\kappa \mathrm{B}$ pathway inhibitor, before TNF- $\alpha$ administration, not only was expression of proinflammatory cytokines attenuated, but also SA- $\beta$-gal activity and expression of senescence markers were reduced (Fig. 6B-F). These results suggest a plausible link between NF- $\kappa \mathrm{B}$ activation and aging. Likewise, in H9c2MT7 cardiomyocytes, we found that TNF- $\alpha$-induced ROS production, NF- $\kappa \mathrm{B}$ activation, proinflammatory cytokine expression, and age-associated phenomena were effectively decreased by MT overexpression (Fig. 6A-F).

Furthermore, TNF- $\alpha$ treatment induced high levels of apoptosis in $\mathrm{H} 9 \mathrm{c} 2$ cardiomyocytes, as demonstrated by increases in the proportion of TUNEL-positive cells (Supplementary Fig. S9A) and caspase-3 activity (Supplementary Fig. S9B). According to both metrics, apoptosis was effectively inhibited 
A

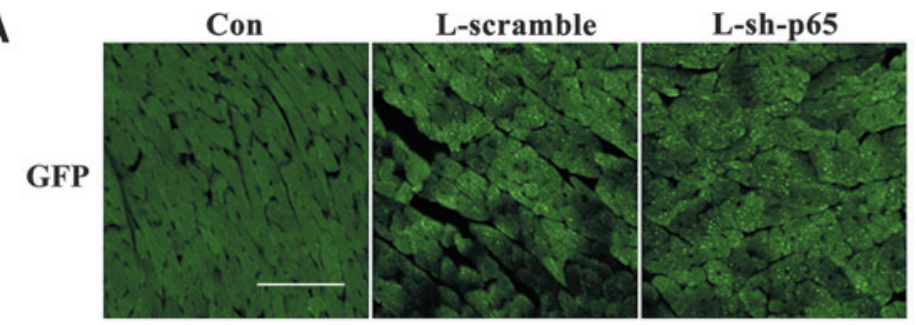

B
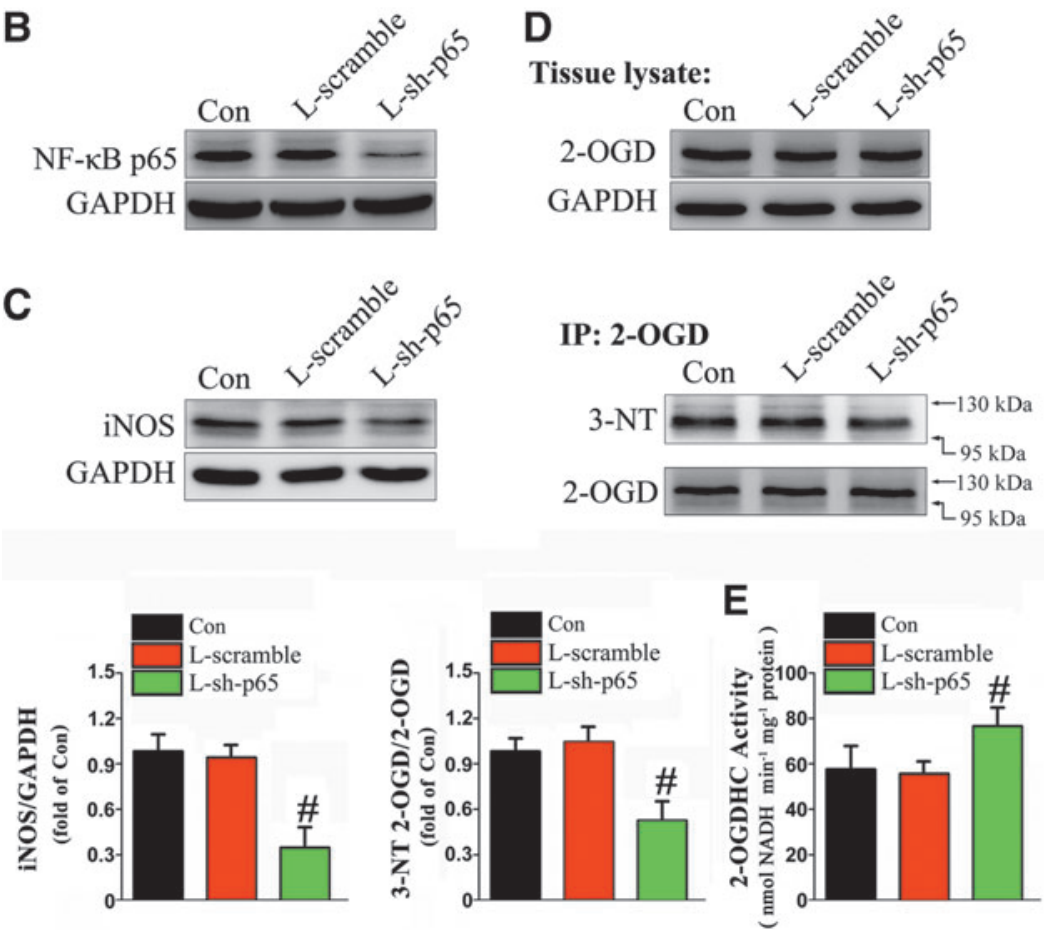

FIG. 5. NF- $\boldsymbol{\kappa}$ B inhibition plays a major role in the attenuation of nitrative damage to 2-OGD. Lentivirus plasmids containing sh-p65 (LRV-U6-p65-CMV-EGFP, L-sh-p65) and a scrambled sequence (LRV-U6-scramble-CMV-EGFP, Lscramble) were directly injected into the myocardium of 15 month-old WT mice over a period of 12 weeks. (A) The expression of EGFP was determined in the frozen heart tissue sections to confirm the localization into the myocardium; $n=6$ for each group. Scale bars $=50 \mu \mathrm{m}$. (B) The expression level of p65 was confirmed in the hearts of lentivirus-treated WT mice by immunoblotting; $n=6$ for each group. (C) Immunoblots of heart homogenates from lentivirus-treated or non-treated WT mice to examine iNOS levels. The histogram (lower panel) is the immunoblotting quantitative analysis of the corresponding immunoblots, and the data are expressed as fold over non-treated control; $n=6$ for each group. (D) Immunoblots of heart homogenates from lentivirus-treated or non-treated WT mice to examine total 2-OGD levels. Then, the heart homogenates were subjected to immunoprecipitation with 2-OGD antibody, followed by immunoblotting with 3-NT and 2-OGD-specific antibody. The histogram (lower panel) is the immunoblotting quantitative analysis of 3-NT 2-OGD/2-OGD, and the data are expressed as fold over non-treated control; $n=6$ for each group. (E) Heart homogenates from lentivirus-treated or non-treated WT mice were subjected to 2-OGDHC activity assay in isolated mitochondria; $n=6$ for each group. Data are presented as mean \pm SD. ${ }^{\#} p<0.05$ versus non-treated mice. 2-OGDHC, 2-oxoglutarate dehydrogenase complex.

by co-treatment with MnTMPyP, urate, or Bay-117082, similar to the effect of MT overexpression in H9c2MT7 cardiomyocytes (Supplementary Fig. S9A, B). Next, we investigated the mechanism underlying TNF- $\alpha$-induced apoptosis and the inhibitory effect of MT.

TNFR1 protein level was not altered, but TNF- $\alpha$-induced apoptosis was shown to be closely linked to the induction of a receptor-mediated downstream signaling cascade, demonstrated by immunoprecipitation of TNFR1-associated death domain (TRADD) with TNFR1 protein (Supplementary Fig. S9C), as well as higher levels of active caspase-8 in $\mathrm{H} 9 \mathrm{c} 2$ cardiomyocytes (Supplementary Fig. S9D). Moreover, the levels of Bax/Bcl-2, mitochondrial Cytochrome $\mathrm{C}$, and active caspase- 9 were significantly elevated after TNF- $\alpha$ treatment of $\mathrm{H} 9 \mathrm{c} 2$ cardiomyocytes, indicating that the mitochondrial apoptotic cascade also plays a role in TNF- $\alpha-$ induced cardiac apoptosis (Supplementary Fig. S9D). On the other hand, the overexpressed MT comprehensively inhibited TNF- $\alpha$-induced apoptosis via both the receptormediated and mitochondrial pathways (Supplementary Fig. S9C, D).

MT prevents $N F-\kappa B$ activation-mediated nitrative damage to 2-OGD, thus preserving the enzyme activity and energy equilibrium in vitro

As NF- $\kappa \mathrm{B}$ activation is connected with age-associated symptoms in cardiomyocytes, the possibility of an NF- $\kappa \mathrm{B}$ - 

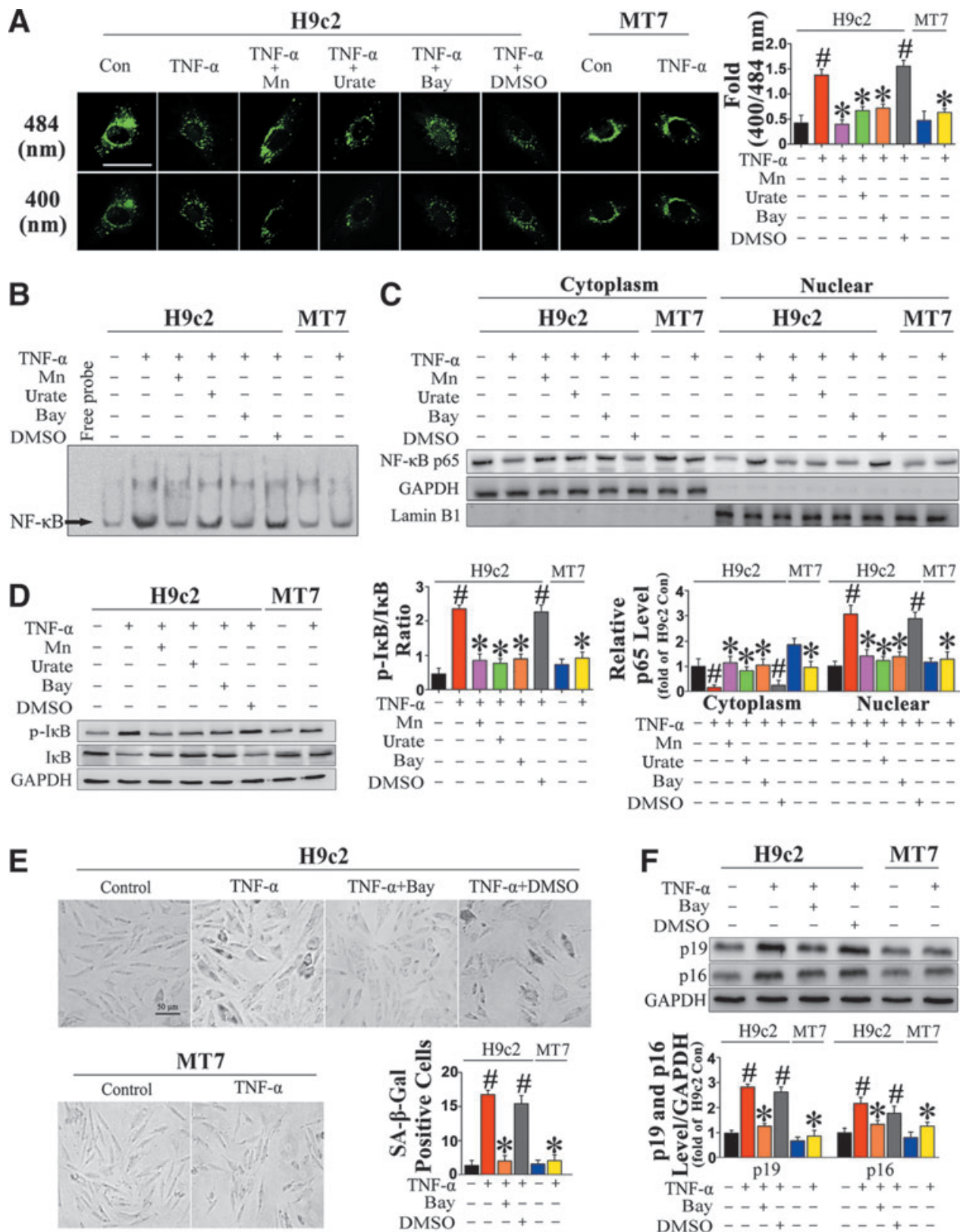

H9c2

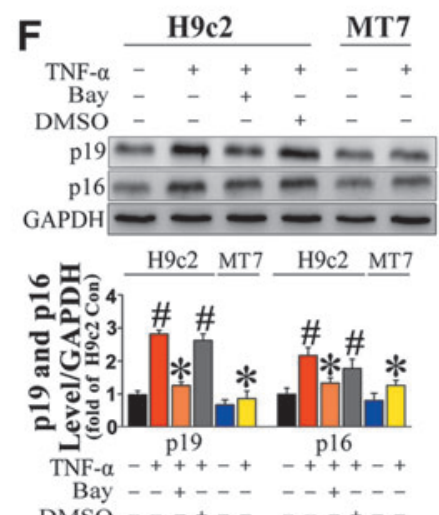

FIG. 6. MT inhibits TNF- $\alpha$-induced NF- $\boldsymbol{k B}$ activation and aging phenotypes in vitro. H9c2 and H9c2MT7 cardiomyocytes were cultured to near (80-90\%) confluence and then administered with TNF- $\alpha$ in a concentration of $100 \mathrm{ng} / \mathrm{ml}$ for $24 \mathrm{~h}$. In addition, $\mathrm{H} 9 \mathrm{c} 2$ cardiomyocytes were co-incubated with $100 \mu M$ urate or $50 \mu M$ MnTMPyP with TNF- $\alpha$. In some experiments, Bay-117082 was pretreated for $10 \mathrm{~min}$ in a concentration of $1.5 \mu M$ before TNF- $\alpha$ administration. (A) H9c2 and H9c2MT7 cardiomyocytes stably expressing cytosolic-localized redox-sensitive GFP mutant (Cyto-RoGFP) were treated under each condition as mentioned earlier. The images are of cells viewed using a confocal microscope; the excitation wavelengths were 400 and $484 \mathrm{~nm}$, and the emission wavelength was $525 \mathrm{~nm}$. The histogram (right panel) is the quantitative analysis of fluorescence intensity at $400 \mathrm{~nm} / 484 \mathrm{~nm}$. For each experimental condition, six different areas were quantified; $n=6$ for each group. (B) Nuclear extracts made from the H9c2 and H9c2MT7 cardiomyocytes under each experimental condition mentioned earlier were subjected to EMSA to measure NF- $\kappa$ B DNA-binding activity, and a representative EMSA gel is presented; $n=6$ for each group. (C) Nuclear and cytosolic extracts were isolated, and the nuclear translocation of p65 was analyzed by immunoblotting. The histogram (lower panel) is the quantitative analysis of the corresponding immunoblots, and the data are expressed as fold over H9c2 control; $n=6$ for each group. (D) Immunoblots of the H9c2 and H9c2MT7 cardiomyocytes under each experimental condition were probed for phosphor- $\mathrm{I} \kappa \mathrm{B} \alpha$ and total $\mathrm{I} \kappa \mathrm{B} \alpha$ expression. The histogram (right panel) is the quantitative result of $\mathrm{pI} \kappa \mathrm{B} \alpha / \mathrm{I} \kappa \mathrm{B} \alpha$ ratio; $n=6$ for each group. (E) H9c2 and H9c2MT7 cardiomyocytes under each experimental condition were analyzed for SA- $\beta$-gal staining. The histogram (lower panel) is the quantitative analysis of SA- $\beta$-gal-positive cells in at least six separate fields; $n=6$ for each group. Scale bars $=100 \mu \mathrm{m}$. $(\mathbf{F})$ The protein levels of cell cycle inhibitors (senescence markers) p19 and p16 were also examined via immunoblotting. The histogram (lower panel) is the quantitative analysis of the corresponding immunoblots, and the data are expressed as fold over H9c2 control; $n=6$ for each group. Data are presented as mean \pm SD. ${ }^{*} p<0.05$ versus $\mathrm{H} 9 \mathrm{c} 2$ control; ${ }^{*} p<0.05$ versus TNF- $\alpha$-treated $\mathrm{H} 9 \mathrm{c} 2$ cardiomyocytes. MnTMPyP, Mn(III) tetrakis 1-methyl 4-pyridyl porphyrin pentachloride. 
mediated increase in the 2-OGD nitration level in TNF- $\alpha$ treated $\mathrm{H} 9 \mathrm{c} 2$ cardiomyocytes was investigated. To this end, we first monitored iNOS protein expression (Fig. 7A) and nitrite production in $\mathrm{H} 9 \mathrm{c} 2$ cardiomyocytes (Supplementary Fig. S3B) after TNF- $\alpha$ treatment. Next, we assessed 3-NT levels in $\mathrm{H} 9 \mathrm{c} 2$ cardiomyocytes after TNF- $\alpha$ administration for
$24 \mathrm{~h}$. Coincidentally, quantitative analysis by immunoblotting revealed an obvious enhancement of 3-NT modification in treated cells, mainly in a protein band at $\sim 115 \mathrm{kDa}$ (Supplementary Fig. S10). The immunoprecipitation verified an increase in 2-OGD nitration level induced by TNF- $\alpha$, without a change in 2-OGD expression (Fig. 7B).
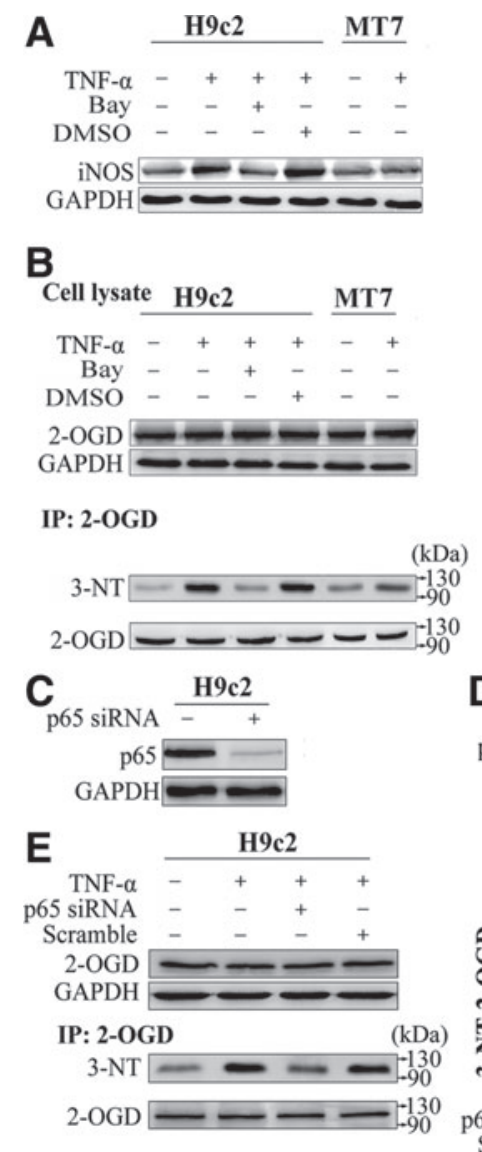
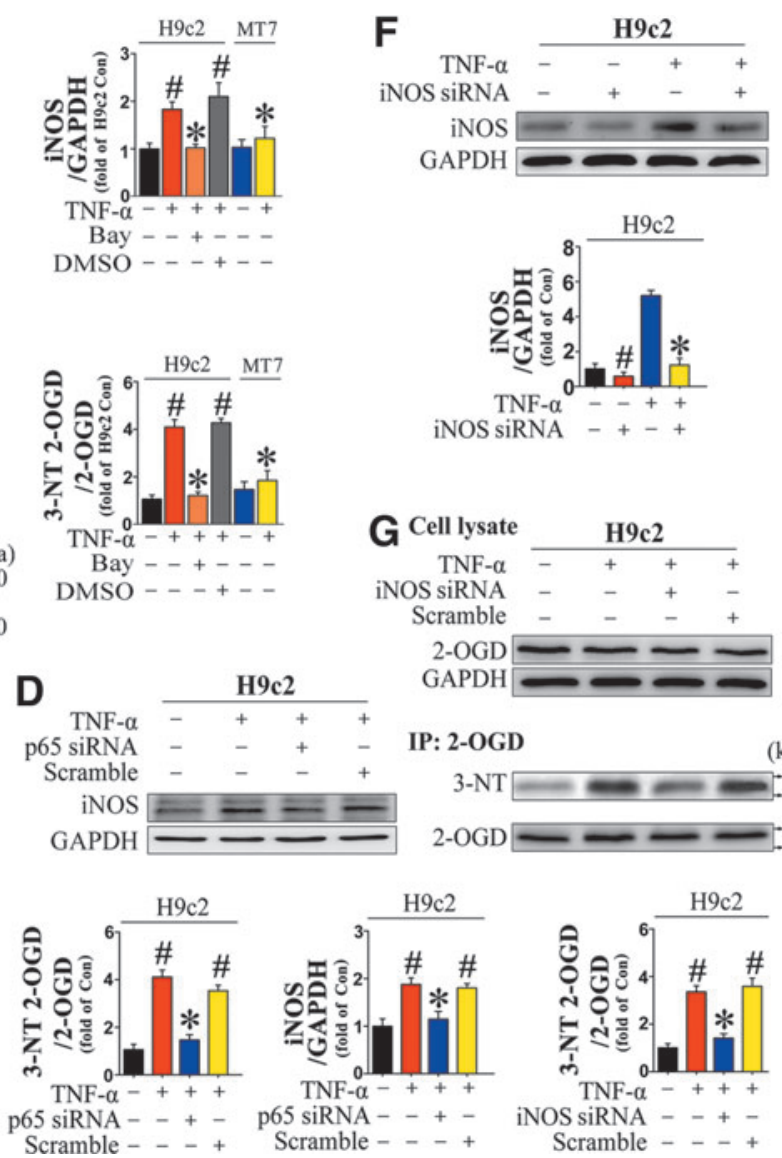

FIG. 7. MT prevents NF- $\boldsymbol{~} \mathbf{B}$ activation-mediated nitrative damage to 2-OGD. For (A, B), H9c2 and H9c2MT7 cardiomyocytes were administered with TNF- $\alpha$ in a concentration of $100 \mathrm{ng} / \mathrm{ml}$ for $24 \mathrm{~h}$. In some experiments, Bay-117082 was pretreated for $10 \mathrm{~min}$ in a concentration of $1.5 \mu \mathrm{M}$ before TNF- $\alpha$ administration. (A) Immunoblots was performed to examine iNOS expression levels. The histogram (right panel) is the immunoblotting quantitative analysis of the corresponding immunoblots, and the data are expressed as fold over $\mathrm{H} 9 \mathrm{c} 2$ control; $n=6$ for each group. (B) Immunoblot analysis of the total 2-OGD expression levels was performed in cell lysates. Then, the cell lysates were subjected to immunoprecipitation with 2OGD antibody, followed by immunoblotting with 3-NT and 2-OGD-specific antibody. The histogram (right panel) is the immunoblotting quantitative analysis of 3-NT 2-OGD/2-OGD, and the data are expressed as fold over H9c 2 control; $n=6$ for each group. For (C-E), H9c2 cardiomyocytes were transfected with p65 siRNA or a scrambled sequence before TNF- $\alpha$ administration. (C) The expression level of p65 was confirmed in the H9c2 cardiomyocytes after p65 siRNA transfection by immunoblotting; $n=6$ for each group. (D) Immunoblots of the transfected or non-treated control H9c2 cardiomyocytes to examine iNOS levels. The histogram (lower panel) is the immunoblotting quantitative analysis of the corresponding immunoblots, and the data are expressed as fold over non-treated control; $n=6$ for each group. (E) Immunoblot analysis of the total 2-OGD expression levels was performed in transfected or non-treated cell lysates. Then, the cell lysates were subjected to immunoprecipitation with 2-OGD antibody, followed by immunoblotting with 3-NT and 2-OGD-specific antibody. The histogram (right panel) is the immunoblotting quantitative analysis of 3-NT 2-OGD/2-OGD, and the data are expressed as fold over non-treated control; $n=6$ for each group. For $(\mathbf{F}, \mathbf{G}), \mathrm{H} 9 \mathrm{c} 2$ cardiomyocytes was transfected with iNOS siRNA or a scrambled sequence before TNF- $\alpha$ administration. (F) The iNOS expression level was confirmed in the H9c 2 cardiomyocytes after iNOS siRNA transfection with or without TNF- $\alpha$ treatment by immunoblotting. The histogram (lower panel) is the immunoblotting quantitative analysis of the corresponding immunoblots, and the data are expressed as fold over non-treated control; $n=6$ for each group. (G) Immunoblot analysis of the total 2-OGD expression levels was performed in transfected or non-treated cell lysates. Then, the cell lysates were subjected to immunoprecipitation with 2-OGD antibody, followed by immunoblotting with 3-NT and 2-OGD-specific antibody. The histogram (right panel) is the immunoblotting quantitative analysis of 3-NT 2-OGD/2-OGD, and the data are expressed as fold over non-treated control; $n=6$ for each group. Data are presented as mean $\pm \mathrm{SD}$. (A, B) ${ }^{\#} p<0.05$ versus $\mathrm{H} 9 \mathrm{c} 2$ control; ${ }^{*} p<0.05$ versus $\mathrm{TNF}-\alpha$-treated $\mathrm{H} 9 \mathrm{c} 2$ cardiomyocytes. (C-G) ${ }^{\#} p<0.05$ versus non-treated control; ${ }^{*} p<0.05$ versus $\mathrm{TNF}-\alpha$-treated $\mathrm{H} 9 \mathrm{c} 2$ cardiomyocytes. 
Consistent with results obtained in vivo, TNF- $\alpha$-induced nitrative damage to 2-OGD also had a significant effect on 2-OGDHC activity, kinetics, and energy charge in H9c2 cardiomyocytes (Supplementary Fig. S11 and Supplementary Table S5). When TNF- $\alpha$-induced NF- $\kappa \mathrm{B}$ activation was restrained in $\mathrm{H} 9 \mathrm{c} 2$ cardiomyocytes by pretreatment with Bay-117082, iNOS expression (Fig. 7A) and nitrite production (Supplementary Fig. S3B) were also effectively reduced, concomitant with a reduction in 2-OGD nitration level (Fig. 7B). Subsequently, 2-OGDHC activity, kinetics, and energy charge were well preserved (Supplementary Fig. S11 and Supplementary Table S5).

The roles of TNF- $\alpha$-induced NF- $\kappa \mathrm{B}$ activation and iNOS upregulation in 2-OGD nitrative damage were further confirmed by gene silencing of p65 and iNOS. For these experiments, H9c2 cardiomyocytes were transfected with p65- or iNOS-specific siRNA for $8 \mathrm{~h}$, followed by TNF- $\alpha$ treatment for $24 \mathrm{~h}$. Transfection of p65 siRNA dramatically suppressed NF- $\kappa$ B p65 expression in H9c2 cardiomyocytes (Fig. 7C). In addition, p65 knockdown abolished TNF- $\alpha$-induced upregulation of proinflammatory cytokines at both the protein and mRNA levels (Supplementary Fig. S12).

The increase of iNOS expression by TNF- $\alpha$ treatment was also effectively inhibited in cells transfected with p65 siRNA (Fig. 7D). Accordingly, TNF- $\alpha$-induced 2-OGD nitrative damage was well prevented (Fig. 7E and Supplementary S13A). Also, a direct inhibitory effect on TNF- $\alpha$-induced 2OGD nitrative damage was achieved by virtue of iNOS siRNA (Fig. 7F, G and Supplementary S13B).

As demonstrated earlier, MT is an effective NF- $\kappa$ B inhibitor in both old mice hearts and TNF- $\alpha$-treated $\mathrm{H} 9 \mathrm{c} 2$ cardiomyocytes. Accordingly, NF- $\kappa \mathrm{B}$-mediated nitrative damage to 2-OGD was less evident in H9c2MT7 cardiomyocytes than in H9c2 cardiomyocytes, which expressed lower levels of iNOS (Fig. 7A), produced less nitrite (Supplementary Fig. S3B), and exhibited less 3-NT modification on 2-OGD on TNF- $\alpha$ treatment (Fig. 7B). In addition, enzyme activity and energy charge were well preserved in H9c2 cardiomyocytes (Supplementary Fig. S11).

SiRNA-mediated knockdown of MT2A overexpressed in H9c2MT7 cardiomyocytes (Fig. 8A) abolished the protective effects against TNF- $\alpha$ treatment, including MT-mediated inhibition of NF- $\kappa$ B DNA binding (Fig. $8 \mathrm{~B}$ ), reduction in nuclear p65 translocation (Fig. 8C), and downregulation of proinflammatory cytokines (Fig. 8D, E). More importantly, the originally observed alleviation of TNF- $\alpha$-induced nitrative damage to 2-OGD was abolished by silencing of MT (Fig. 8F, G).

Considering that the MT-elicited effects are mainly due to its role in iron chelating (38), we next investigated whether similar results could be obtained by other iron chelators. Therefore, a selective iron chelator-desferrioxamine mesylate (DFO) (10) was pretreated in H9c2 cardiomyocytes before TNF- $\alpha$ administration. The results showed that DFO pretreatment could suppress TNF- $\alpha$-induced NF- $\kappa$ B activation and the following nitrative damage to 2-OGD, to some extent, but the inhibitory effects exerted by DFO were slighter than those of MT overexpression (Supplementary Fig. S14).

\section{Identification and quantitation of nitrated peptides of 2-OGD}

Since nitration of 2-OGD is involved in the process of aging, details regarding nitration of this protein need to be explored. As such, LC-MS was introduced for site mapping and quantitation of the nitrated peptides of 2-OGD.

For a full investigation of all nitration sites of 2-OGD, a parallel-reaction monitoring (PRM)-targeted MS/MS method was utilized to conduct analysis in old WT mice hearts, and eight 3-NT-modified peptides of 2-OGD were successfully identified with a high confidence level. The representative mass spectra of identified 3-NT-modified peptides are shown in Supplementary Figure S15.

After PRM, the confirmed 3-NT-modified peptides and their non-nitrated counterparts were defined as targets for further quantitation of the nitration level. We used a selective reaction monitoring (SRM)-based targeted proteomics method to quantitate relative differences in the degree of 2OGD nitration in WT and MT-TG (MM, MT-1) mice hearts at the three age stages defined earlier (young, middle-aged, and old). The SRM results revealed that $\mathrm{Y}^{175}, \mathrm{Y}^{460}, \mathrm{Y}^{910}$, $\mathrm{Y}^{965}, \mathrm{Y}^{966}, \mathrm{~W}^{984}$, and $\mathrm{Y}^{968}$ were significantly nitrated in old WT mice, but these modifications were downregulated in old MT-TG mice (Fig. 9A-H i).

Computational models illustrated that the tyrosine sites identified earlier are located on the surface of 2-OGD, making them more sensitive to nitration (Fig. 9A-H ii, iii). The SRM results of $\mathrm{Y}^{658}$ displayed a relatively high nitration degree in all the three age stages without an obvious agedependent increase in nitration level. In addition, MT did not significantly protect this residue against nitration (Fig. 9C i). Three-dimensional structural analysis revealed that $\mathrm{Y}^{658}$ is located in the interior of 2-OGD (Fig. 9C ii, iii), which may explain these observations.

To confirm the results obtained in vivo, the same PRM strategy was performed on TNF- $\alpha$-treated cardiomyocytes. Seven nitration sites were detected with high confidence (Table 1). In addition, a quantitative analysis of the nitration degree for each peptide was also performed (Supplementary Fig. S16). The in vitro results were, to some extent, coincident with the observations in old mice hearts.

\section{Discussion}

The data here establish that MT overexpression in the heart largely decreases tyrosine nitration levels in this model organism; dramatically alleviates myocardial hypertrophy, dysfunction, and remodeling; and postpones the occurrence of senescence phenotypes. In addition, in the course of our experiments, we found that old mice exhibit elevated NF- $\kappa \mathrm{B}$ activity and a resultant chronic inflammation response, which are considered critical in the development of numerous CVD during aging (20). Accordingly, in this study, we observed several senescence-associated phenotypes, such as elevated SA- $\beta$-gal activity and upregulation of senescence markers $\left(\mathrm{p} 16^{\mathrm{INK} 4 \mathrm{a}}\right.$ and $\left.\mathrm{p} 19^{\mathrm{ARF}}\right)$, in TNF- $\alpha$-treated H9c2 cardiomyocytes. These phenotypes were abolished when NF- $\kappa \mathrm{B}$ activation was blocked, consistent with the notion of a link between inflammation and aging.

$\mathrm{NF}-\kappa \mathrm{B}$ activation during aging is likely to be responsible for the increased levels of adhesion molecules and iNOS in old mice hearts (14), which is considered the main cause of nitration in tyrosine-containing proteins. Although our results demonstrated that MT suppressed NF- $\kappa \mathrm{B}$ activation by the inhibition of $\mathrm{I} \kappa \mathrm{B}$ phosphorylation and further degradation in the cytoplasm in both old mice hearts and TNF- $\alpha$-treated 
A

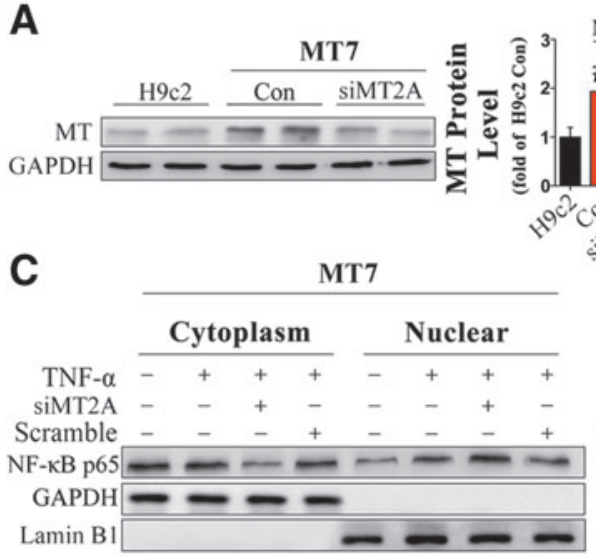

E $_{\text {TNF- } \alpha} \frac{\text { MT7 }}{-+++}$ F

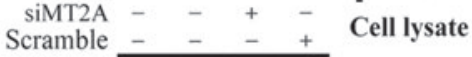
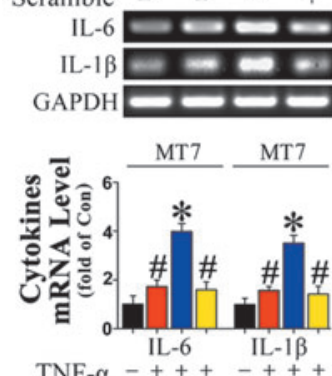

TNF- $\alpha-+++-+++$

siMT2A --+---+

Scramble - - + - - +

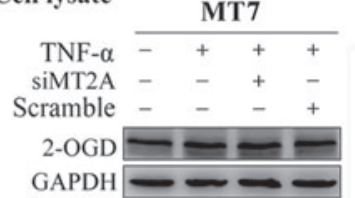

IP: 2-OGD

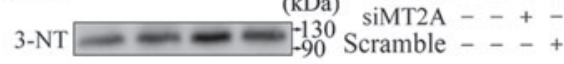

(kDa)

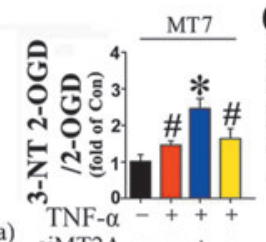

siMT2A - -+

2-OGD $=--130$

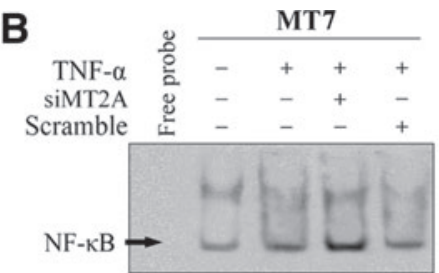

D

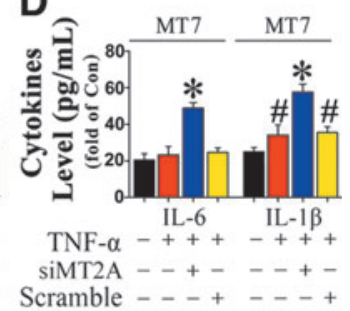

FIG. 8. The protective effects of MT on NF- $\kappa \mathrm{B}$ activation-mediated nitrative damage to 2-OGD are abolished by MT knockdown in H9c2MT7 cardiomyocytes. The H9c2MT7 cardiomyocytes were transfected with specific MT2A siRNA or a scrambled sequence before TNF- $\alpha$ administration. (A) The MT expression level in H9c2, H9c2MT7, and MT2A siRNA transfected H9c2MT7 cardiomyocytes was confirmed. The histogram (right panel) is the immunoblotting quantitative analysis of the corresponding immunoblots, and the data are presented as fold over H9c2 control; $n=6$ for each group. ${ }^{\#} p<0.05$ versus $\mathrm{H} 9 \mathrm{c} 2$ control; ${ }^{\&} p<0.05$ versus $\mathrm{H} 9 \mathrm{c} 2 \mathrm{MT} 7$ cardiomyocytes. (B) Nuclear extracts made from the transfected H9c2MT7 cardiomyocytes under each experimental condition mentioned earlier were subjected to EMSA to measure NF- $\kappa$ B DNA-binding activity, and a representative EMSA gel is presented; $n=6$ for each group. (C) Nuclear and cytosolic extracts were isolated, and the nuclear translocation of p65 was analyzed by immunoblotting. The histogram (right panel) is the quantitative analysis of the corresponding immunoblots, and the data are expressed as fold over non-treated H9c2MT7 control; $n=6$ for each group. (D) The concentrations of IL-6 and IL-1 $\beta$ in the culture medium under each experimental condition were investigated by ELISA; $n=6$ for each group. (E) The transcription levels of IL-6 and IL-1 $\beta$ were determined by sqRT-PCR. The histogram (lower panel) is the quantitative analysis of the corresponding sqRT-PCR, and the data are expressed as fold over non-treated H9c2MT7 control; $n=6$ for each group. (F) Immunoblot analysis of the total 2-OGD expression levels was performed in H9c2MT7 cardiomyocytes under each experimental condition. Then, the cell lysates were subjected to immunoprecipitation with 2-OGD antibody, followed by immunoblotting with 3-NT and 2OGD-specific antibody. The histogram (right panel) is the immunoblotting quantitative analysis of 3-NT 2-OGD/2-OGD, and the data are expressed as fold over non-treated H9c2MT7 control; $n=6$ for each group. (G) Cell lysates from transfected or non-treated H9c2MT7 cardiomyocytes were subjected to 2-OGDHC activity assay in isolated mitochondria; $n=6$ for each group. Data are presented as mean \pm SD. For figures in $\mathrm{B}-\mathrm{G},{ }^{*} p<0.05$ versus $\mathrm{H} 9 \mathrm{c} 2 \mathrm{MT} 7$ control; ${ }^{*} p<0.05$ versus $\mathrm{TNF}-$ $\alpha$-treated H9c2MT7 cardiomyocytes. sqRT-PCR, semi-quantitative RT-PCR.

$\mathrm{H} 9 \mathrm{c} 2$ cardiomyocytes, it remains possible that MT regulates $\mathrm{NF}-\kappa \mathrm{B}$ activity even in the nucleus.

Consistent with this idea, an EMSA supershift analysis was utilized and it revealed that MT directly combines with NF$\kappa \mathrm{B}$ (1). In addition, MT was demonstrated to bind $\mathrm{Zn}$ and regulate cellular Zn homeostasis, which is essential for NF$\kappa \mathrm{B}$ DNA-binding activity $(28,43)$. The results from this work and other studies suggest that, although by different means, $\mathrm{MT}$ acts as a negative regulator of NF- $\kappa \mathrm{B}$ activity. Accordingly, NF- $\kappa$ B-dependent upregulation of iNOS, as well as the resultant nitrative damage to certain proteins, could be attenuated by MT overexpression.

Mass spectrometry analysis revealed that the most significant nitrated protein identified in the old mice hearts is 2OGD, which is the E1 subunit of 2-OGDHC. It is reported that 2-OGDHC is a crucial intracellular target for ROS and plays an important role in the bioenergetic shortage on account of oxidative stress. On the other hand, recent evidence has shown that the enzyme itself is able to generate ROS, and, therefore, it could further enhance the extent of intracellular 


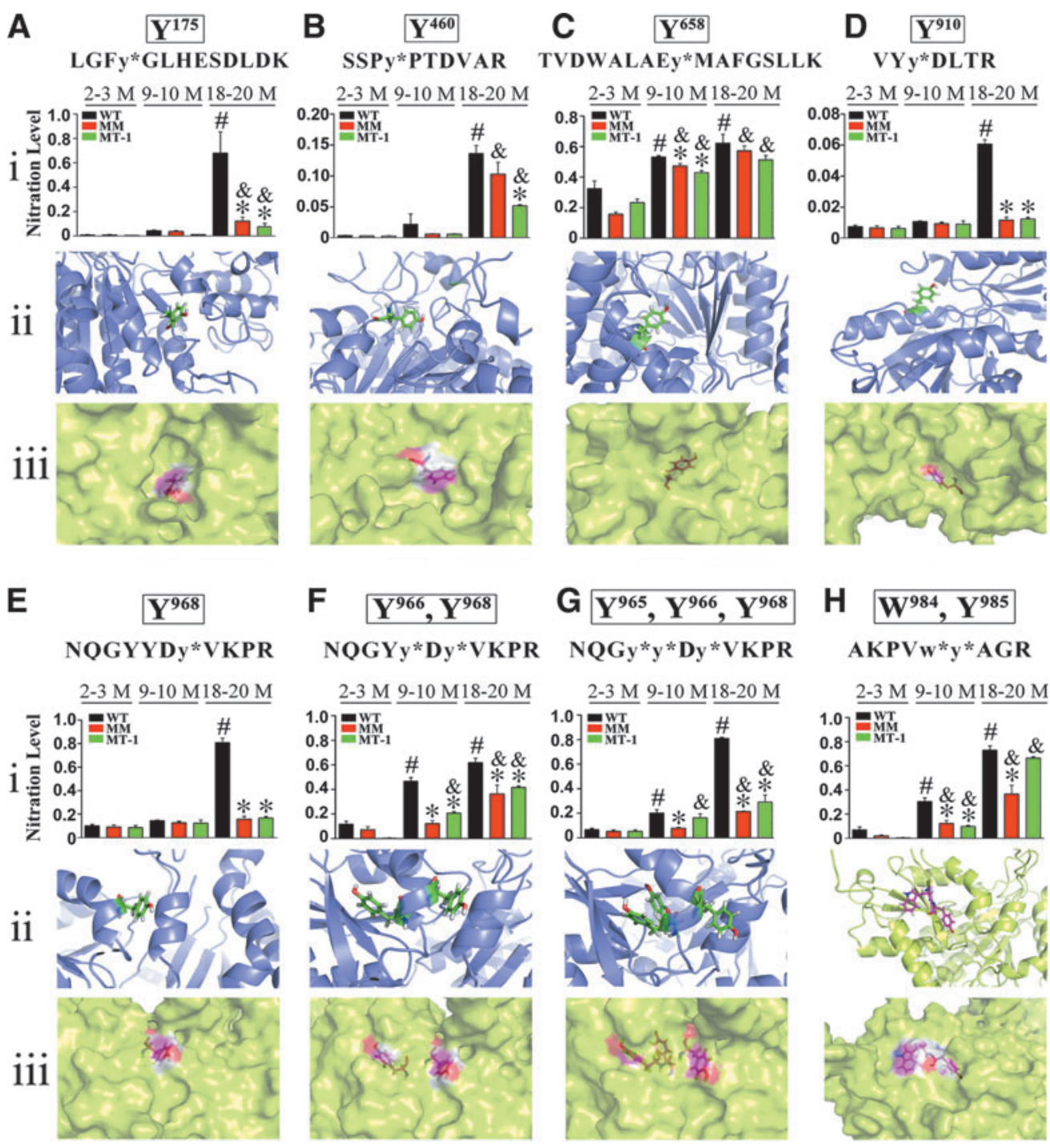

FIG. 9. Quantitative and structural analysis of the identified nitrated peptides in 2-OGD during aging process. The heart homogenates from WT and MT-TG (MM, MT-1) mice at three representative stages (2-3, 9-10, and 18-20 months) were processed with in-gel digestion for LC-MS/MS analysis. (A-H i) The SRM chromatograms for each nitrated peptide were used to evaluate the nitration level of the corresponding peptide; $n=6$ for each group. Data are presented as mean \pm SD. ${ }^{\#} p<0.05$ versus WT mice at $2-3$ months old; $* p<0.05$ versus WT mice in the same age stage; ${ }^{*} p<0.05$ versus the same genotype of MT-TG mice at 2-3 months old. (A-H ii, iii) Protein homology modeling shows a ribbon diagram (ii) and surfacefilled model (iii) of mouse 2-OGD using the 3-D structure 2JGD chain A as a template. SRM, selective reaction monitoring.

oxidative stress (41). Considering the susceptibility of 2OGDHC to oxidative stress, it can be presumed that when the radicals are produced by the enzyme near the ROS-sensitive components, the enzyme itself may also be inactivated. Therefore, the dual role of the 2-OGDHC as both a target and a generator of ROS may result in its preferential nitration during the aging process.

To date, the majority of studies regarding 2-OGDHC concentrated on revealing the relationship between its inactivation and age-related neurodegenerative diseases $(35,22)$.

Table 1. Nitrated Peptides of 2-OGD in TNF- $\alpha$-Treated H9C2 Cardiomyocytes IDENTIFIED BY SEQUEST HT NODE OF PD 1.4

\begin{tabular}{lccc}
\hline Peptide & Confidence level & Charge & Nitration site \\
\hline K.LGFy*GLHESDLDK.V & High & +2 & $\mathrm{Y}_{175}$ \\
R.SSPy*PTDVAR.V & High & +2 & $\mathrm{Y}_{460}$ \\
R.TVDWALAEy*MAFGSLLK.E & High & +2 & $\mathrm{Y}_{658}$ \\
K.NQGy*y*Dy*VKPR.L & High & +2 & $\mathrm{Y}_{965}, \mathrm{Y}_{966}, \mathrm{Y}_{968}$ \\
R.AKPV $\boldsymbol{w}^{*} \boldsymbol{y}$ *AGR.D & High & +2 & $\mathrm{~W}_{984}, \mathrm{Y}_{985}$ \\
\hline
\end{tabular}

2-OGD, 2-oxoglutarate dehydrogenase; PD, Proteome Discoverer; TNF- $\alpha$, tumor necrosis factor- $\alpha$. 
Nevertheless, the phenomenon and influence of 2-OGDHC inactivation by $\mathrm{ONOO}\urcorner$ in age-dependent CVD has been rarely discussed.

In this study, a PRM method was used to identify the actual nitrated peptides of 2-OGD. Eight nitrated peptides of 2OGD were successfully identified with high confidence, involving eight nitration sites: $\mathrm{Y}^{175}, \mathrm{Y}^{460}, \mathrm{Y}^{658}, \mathrm{Y}^{910}, \mathrm{Y}^{965}$, $\mathrm{Y}^{966}, \mathrm{~W}^{984}$, and $\mathrm{Y}^{968}$. Among these, $\mathrm{Y}^{175}$ is located in the Nterminus of 2-OGD. A previous report demonstrated that 2OGD, the E1 component of the 2-OGDHC, interacts with the E2 core through the N-terminal domain, which consists of a columnar arrangement of four helices formed by residues 85 190 (21). Therefore, we speculate that the nitration of $\mathrm{Y}^{175}$ may directly influence the binding efficiency of 2-OGD to the E2 component. $\mathrm{Y}^{460}$ is located in a highly conserved region shared by many dehydrogenase enzymes (Pfam), and $\mathrm{Y}^{658}$ resides in the transketolase-like, pyrimidine-binding domain formed by residues 649-866.

Therefore, we hypothesize that nitration of these sites exerts significant influence on the function of the enzyme. In parallel, the same PRM strategy was used to analyze the nitration sites of 2-OGD in TNF- $\alpha$-treated cardiomyocytes. The results revealed that NF- $\kappa$ B activation-mediated 2-OGD nitration at $\mathrm{Y}^{175}, \mathrm{Y}^{460}, \mathrm{Y}^{910}, \mathrm{Y}^{965}, \mathrm{Y}^{966}, \mathrm{~W}^{984}$, and $\mathrm{Y}^{968}$ is highly concordant with the nitration sites observed in old mice hearts. Therefore, we speculate that the physiological processes of aging and inflammation may share some commonalities, resulting in similar nitrative damage to the enzyme.

In addition to nitration on tyrosine, a reversible modification on the cysteine of 2-OGD has also been reported to have influence on the catalytic activity of 2-OGDHC (33). Considering that the thiol-containing cysteine in redoxsensitive proteins is particularly sensitive to alterations in the redox environment $(9,16)$, the possible role of cysteine oxidation in 2-OGD during cardiac aging was assayed. Snitrosation, a representative reversible modification on the cysteine caused by NO hyperproduction (45), was detected by the biotin-switch method. The results revealed no apparent distinction of S-nitrosation in 2-OGD between young and old WT mice hearts. Besides, the irreversible cysteine oxidation in 2-OGD (sulfinic and sulfonic acid formation) was also assessed by MS analysis. However, only tyrosine nitration in 2-OGD was detected in the old WT mice hearts.

The present study revealed that the activity of 2-OGDHC has suffered $\sim 30 \%$ diminishment in both old mice hearts and TNF- $\alpha$-treated cardiomyocytes. In addition, alterations in KGDHC kinetic characteristics (elevated $K_{\mathrm{m}}$ and decreased $V_{\max }$ ) indicated a reduced affinity for the substrate and slower catalysis. Such a dysfunction of 2-OGDHC has major functional implications considering that the 2OGDHC-catalyzed reaction is significant for energy production as well as for the metabolic interaction between mitochondria and cytoplasm.

Perturbation of these functions might link 2-OGDHC inactivation and cardiac degenerative diseases, mainly through (i) decreased energy production, especially in energyconsuming organs, such as the heart. Decreased ATP production resulting from 2-OGDHC inactivation in the heart was associated with an exacerbation of cardiac function, as shown by a reduction in FS\% and LV hypertrophy (30), and (ii) changes in the normal flow of reducing equivalents and metabolites among cellular compartments (11).
These hypotheses are, indeed, supported by observing a reduction of the total ATP level and NADH content in isolated mitochondria from both old mouse hearts and TNF- $\alpha$ treated cardiomyocytes. Moreover, our detailed analysis of variation in adenosine nucleotides via ion-pairing LC-MS revealed a decrease of energy charge in both old mice hearts and TNF- $\alpha$-treated cardiomyocytes $(\sim 16 \%$ and $\sim 28 \%$, respectively). Taken together, the role of 2-OGDHC in overall metabolism renders this enzyme a promising target for metabolic regulation in various kinds of age-related degenerative diseases.

In summary, our findings demonstrate that the protective effects of MT on age-associated CVD can be attributed mainly to its role in NF- $\kappa \mathrm{B}$ inhibition and the resultant alleviation of nitrative damage. Moreover, we revealed that 2-OGD, as the E1 component of 2-OGDHC, was preferentially nitrated during inflamm-aging. The nitration of 2-OGD was shown to correlate with the diminution of 2-OGDHC catalytic function and the reduction of energy charge. This novel finding provides a possible insight into the mechanisms by which it might act through alleviating the nitration of the enzyme involved in intermediate metabolism during the aging process, MT preserves the normal function of the enzyme, thus protecting the heart against age-associated cardiomyopathy.

To our knowledge, this is the first study to report these nitration sites on 2-OGD from the standpoint of aging. Further studies are needed to elaborate a causative role of 2-OGD nitration in age-associated CVD by virtue of in vitro expression systems and site-directed mutagenesis.

\section{Materials and Methods}

\section{Animals}

The MT-TG mice were first generated by Kang et al. (25). MT-1, cardiac-specific overexpressing MT about 80-fold that of normal mice; MM, cardiac-specific overexpressing MT about 10-fold that of normal mice, as shown in Supplementary Figure S17. These two lines of MT-TG mice were gifted by Dr. Lu Cai (Department of Pediatrics, University of Louisville). Briefly, MT-TG mice were reproduced from FVB mice, and they have been well characterized in the literature $(12,13,37)$. Both MT-TG-positive mice (heterozygotes) and negative littermates (WT) were kept in the same cage for 4 weeks from birth and then kept in separate, but identical cages.

Based on the standardized protocol, all mice were housed at a stable room temperature with a $12 \mathrm{~h}$ light/dark cycle and offered ad libitum access to food and water. MT-TG and WT mice littermates at three representative age stages $(2-3,9-10$, and 18-20 months) were euthanized without fasting and analyzed. All animal experiments and methods were approved by the Animal Policy and Welfare Committee of Wenzhou Medical University, Wenzhou, Zhejiang Province, China.

\section{Cell cultures}

H9c2 and H9c2MT7 (stable overexpression of human MT2A) rat cardiomyocytes were purchased from American Type Cell Collection (ATCC) and cultured using Dulbecco's modified Eagle's medium (DMEM) (Mediatech, Inc.) that was supplemented with $10 \%$ fetal bovine serum (Invitrogen). $\mathrm{H} 9 \mathrm{c} 2$ and H9c2MT7 cardiomyocytes in all the experiments 
were in passages between 3 and 15 (39). The H9c2 and H9c2MT7 cardiomyocytes were incubated using different concentrations of TNF- $\alpha$ (PreproTech, Rocky Hill, NJ), and a final concentration of $100 \mathrm{ng} / \mathrm{ml}$ of TNF- $\alpha$ was added for $24 \mathrm{~h}$ of incubation. This concentration was selected because of its explicit effect on inducing iNOS in cardiomyocytes $(4,36)$ compared with other concentrations (31).

In some experiments, H9c 2 cardiomyocytes were also coincubated with either $100 \mu \mathrm{M}$ of $\mathrm{ONOO}^{\urcorner}$scavenger urate (Sigma) or $50 \mu M$ MnTMPyP (Calbiochem) plus TNF- $\alpha$. The influence of TNF- $\alpha$-induced NF- $\kappa \mathrm{B}$ activation on cardiomyocytes was defined by pretreating Bay-117082, a representative NF- $\kappa \mathrm{B}$ pathway inhibitor, for $10 \mathrm{~min}$ in a concentration of $1.5 \mu M$ before TNF- $\alpha$ administration.

For determining the influence of iron chelators on TNF- $\alpha$ induced effects, H9c2 cardiomyocytes were pretreated for $30 \mathrm{~min}$ with or without $10 \mathrm{~m} M$ DFO (5) before $100 \mathrm{ng} / \mathrm{ml}$ $\mathrm{TNF}-\alpha$ administration.

\section{Statistical analysis}

Prism 5 software (GraphPad) was utilized for statistical analysis. All data were presented as mean \pm standard deviation. Comparison between two groups was analyzed by independent $t$-tests. Comparisons among multiple groups were analyzed by one-way analysis of variance (ANOVA) or twoway ANOVA, as appropriate. A two-tailed $p$-value less than 0.05 was considered statistically significant.

More methods and materials were provided in Supplementary Data.

\section{Acknowledgments}

This work was supported by grants from the National Nature Science Foundation of China (81371753, 81300171, 81570368, 81500295, and 81470061), the Zhejiang Provincial Natural Science Foundation (LQ14H070002), the Technology Program of Wenzhou (Y20150010), the Public Projects of Zhejiang Province (2015C37064), and the College Students' Technological Innovation Activities of Zhejiang Province (2014R413080).

\section{Author Disclosure Statement}

No competing financial interests exist.

\section{References}

1. Abdel-Mageed AB and Agrawal KC. Activation of nuclear factor kappaB: potential role in metallothionein-mediated mitogenic response. Cancer Res 58: 2335-2338, 1998.

2. Ahmed EK, Picot CR, Bulteau AL, and Friguet B. Protein oxidative modifications and replicative senescence of WI38 human embryonic fibroblasts. Ann N Y Acad Sci 1119: 88-96, 2007.

3. Ahmed EK, Rogowska-Wrzesinska A, Roepstorff P, Bulteau AL, and Friguet B. Protein modification and replicative senescence of WI-38 human embryonic fibroblasts. Aging Cell 9: 252-272, 2010.

4. Aikawa R, Nitta-Komatsubara Y, Kudoh S, Takano H, Nagai T, Yazaki Y, Nagai R, and Komuro I. Reactive oxygen species induce cardiomyocyte apoptosis partly through TNF-alpha. Cytokine 18: 179-183, 2002.

5. Autelli R, Crepaldi S, De Stefanis D, Parola M, Bonelli G, and Baccino FM. Intracellular free iron and acidic path- ways mediate TNF-induced death of rat hepatoma cells. Apoptosis 10: 777-786, 2005.

6. Baraibar MA, Barbeito AG, Muhoberac BB, and Vidal R. A mutant light-chain ferritin that causes neurodegeneration has enhanced propensity toward oxidative damage. Free Radic Biol Med 52: 1692-1697, 2012.

7. Baraibar MA, Liu L, Ahmed EK, and Friguet B. Protein oxidative damage at the crossroads of cellular senescence, aging, and age-related diseases. Oxid Med Cell Longev 2012; DOI: $10.1155 / 2012 / 919832$.

8. Bokov A, Chaudhuri A, and Richardson A. The role of oxidative damage and stress in aging. Mech Ageing Dev 125: 811-826, 2004.

9. Brandes N, Schmitt S, and Jakob U. Thiol-based redox switches in eukaryotic proteins. Antioxid Redox Signal 11: 997-1014, 2009.

10. Brewer GJ. Iron and copper toxicity in diseases of aging, particularly atherosclerosis and Alzheimer's disease. Exp Biol Med (Maywood) 232: 323-335, 2007.

11. Bunik VI and Fernie AR. Metabolic control exerted by the 2-oxoglutarate dehydrogenase reaction: a cross-kingdom comparison of the crossroad between energy production and nitrogen assimilation. Biochem J 422: 405-421, 2009.

12. Cai L, Wang J, Li Y, Sun X, Wang L, Zhou Z, and Kang YJ. Inhibition of superoxide generation and associated nitrosative damage is involved in metallothionein prevention of diabetic cardiomyopathy. Diabetes 54: 1829-1837, 2005.

13. Cai L, Wang Y, Zhou G, Chen T, Song Y, Li X, and Kang YJ. Attenuation by metallothionein of early cardiac cell death via suppression of mitochondrial oxidative stress results in a prevention of diabetic cardiomyopathy. J Am Coll Cardiol 48: 1688-1697, 2006.

14. Csiszar A, Ungvari Z, Edwards JG, Kaminski PM, Wolin MS, Koller A, and Kaley G. Aging-induced phenotypic changes and oxidative stress impair coronary arteriolar function. Circ Res 90: 1159-1166, 2002.

15. Csiszar A, Wang M, Lakatta EG, and Ungvari Z. Inflammation and endothelial dysfunction during aging: role of NF- $\kappa$ B. J Appl Physiol 105: 1333-1341, 2004.

16. Cook KM and Hogg PJ. Post-translational control of protein function by disulfide bond cleavage. Antioxid Redox Signal 18: 1987-2015, 2013.

17. Dutsch-Wicherek M, Sikora J, and Tomaszewska R. The possible biological role of metallothionein in apoptosis. Front Biosci 13: 4029-4038, 2008.

18. Esberg LB and Ren J. Role of nitric oxide, tetrahydrobiopterin and peroxynitrite in glucose toxicityassociated contractile dysfunction in ventricular myocytes. Diabetologia 46: 1419-1427, 2003.

19. Ferrini M, Magee TR, Vernet D, Rajfer J, and GonzálezCadavid NF. Aging-related expression of inducible nitric oxide synthase and markers of tissue damage in the rat penis. Biol Reprod 64: 974-982, 2001.

20. Franceschi $C$, Bonafè $M$, Valensin $S$, Olivieri F, De Luca M, Ottaviani E, and De Benedictis G. Inflamm-aging. An evolutionary perspective on immunosenescence. Ann $N Y$ Acad Sci 908: 244-254, 2000.

21. Frank RA, Price AJ, Northrop FD, Perham RN, and Luisi BF. Crystal structure of the E1 component of the Escherichia coli 2-oxoglutarate dehydrogenase multienzyme complex. J Mol Biol 368: 639-651, 2007.

22. Gibson GE, Kingsbury AE, Xu H, Lindsay JG, Daniel S, Foster OJ, Lees AJ, and Blass JP. Deficits in a tricarboxylic 
acid cycle enzyme in brains from patients with Parkinson's disease. Neurochem Int 43: 129-135, 2003.

23. Helenius M, Hänninen M, Lehtinen SK, and Salminen A. Aging-induced up-regulation of nuclear binding activities of oxidative stress responsive NF-kB transcription factor in mouse cardiac muscle. J Mol Cell Cardiol 28: 487-498, 1996.

24. Inoue K, Takano H, Shimada A, and Satoh M. Metallothionein as an anti-inflammatory mediator. Mediators Inflamm 2009: 101659, 2009.

25. Kang YJ, Chen Y, Yu A, Voss-McCowan M, and Epstein PN. Overexpression of metallothionein in the heart of transgenic mice suppresses doxorubicin cardiotoxicity. $J$ Clin Invest 100: 1501-1506, 1997.

26. Krishnamurthy J, Torrice C, Ramsey MR, Kovalev GI, AlRegaiey K, Su L, and Sharpless NE. Ink4a/Arf expression is a biomarker of aging. J Clin Invest 114: 1299-1307, 2004.

27. Lakatta EG and Levy D. Arterial and cardiac aging: major shareholders in cardiovascular disease enterprises, part I: aging arteries: a "set up" for vascular disease. Circulation 107: 139-146, 2003.

28. Lazo JS and Pitt BR. Metallothioneins and cell death by anticancer drugs. Annu Rev Pharmacol Toxicol 35: 635653, 1995.

29. Li SY, Du M, Dolence EK, Fang CX, Mayer GE, Ceylan-Isik AF, LaCour KH, Yang X, Wilbert CJ, Sreejayan N, and Ren J. Aging induces cardiac diastolic dysfunction, oxidative stress, accumulation of advanced glycation end products and protein modification. Aging Cell 4: 57-64, 2005.

30. Mariappan N, Elks CM, Sriramula S, Guggilam A, Liu Z, Borkhsenious $\mathrm{O}$, and Francis J. NF- $\kappa \mathrm{B}$-induced oxidative stress contributes to mitochondrial and cardiac dysfunction in type II diabetes. Cardiovasc Res 85: 473-483, 2010.

31. Müller-Werdan U, Schumann H, Fuchs R, Reithmann C, Loppnow H, Koch S, Zimny-Arndt U, He C, Darmer D, Jungblut P, Stadler J, Holtz J, and Werdan K. Tumor necrosis factor alpha (TNF alpha) is cardiodepressant in pathophysiologically relevant concentrations without inducing inducible nitric oxide-(NO)-synthase (iNOS) or triggering serious cytotoxicity. J Mol Cell Cardiol 29: 2915-2923, 1997.

32. North BJ and Sinclair DA. The intersection between aging and cardiovascular disease. Circ Res 110: 1097-1108, 2012.

33. Nulton-Persson AC, Starke DW, Mieyal JJ, and Szweda LI. Reversible inactivation of alpha-ketoglutarate dehydrogenase in response to alterations in the mitochondrial glutathione status. Biochemistry 42: 4235-4242, 2003.

34. Prieur A and Peeper DS. Cellular senescence in vivo: a barrier to tumorigenesis. Curr Opin Cell Biol 20: 150-155, 2008.

35. Shi Q, Xu H, Yu H, Zhang N, Ye Y, Estevez AG, Deng H, and Gibson GE. Inactivation and reactivation of the mitochondrial $\alpha$-ketoglutarate dehydrogenase complex. J Biol Chem 286: 17640-17648, 2011.

36. Song W, Lu X, and Feng Q. Tumor necrosis factor-alpha induces apoptosis via inducible nitric oxide synthase in neonatal mouse cardiomyocytes. Cardiovasc Res 45: 595$602,2000$.

37. Song Y, Wang J, Li Y, Du Y, Arteel GE, Saari JT, Kang YJ, and Cai L. Cardiac metallothionein synthesis in streptozotocin-induced diabetic mice, and its protection against diabetes-induced cardiac injury. Am J Pathol 167: 17-26, 2005.

38. Swindell WR. Metallothionein and the biology of aging. Ageing Res Rev 10: 132-145, 2011.
39. Tan $Y$, Li X, Prabhu SD, Brittian KR, Chen Q, Yin $X$, McClain CJ, Zhou Z, and Cai L. Angiotensin II plays a critical role in alcohol-induced cardiac nitrative damage, cell death, remodeling, and cardiomyopathy in a protein kinase C/nicotinamide adenine dinucleotide phosphate oxidasedependent manner. J Am Coll Cardiol 59: 1477-1486, 2012.

40. Torres J, Enríquez-de-Salamanca A, Fernández I, Rodríguez-Ares MT, Quadrado MJ, Murta J, Benítez del Castillo JM, Stern ME, and Calonge M. Activation of MAPK signaling pathway and NF- $\kappa \mathrm{B}$ activation in pterygium and ipsilateral pterygium-free conjunctival specimens. Invest Ophthalmol Vis Sci 52: 5842-5852, 2011.

41. Tretter L and Adam-Vizi V. Alpha-ketoglutarate dehydrogenase: a target and generator of oxidative stress. Philos Trans $R$ Soc Lond B Biol Sci 360: 2335-2345, 2005.

42. Yang B and Rizzo V. TNF-alpha potentiates protein-tyrosine nitration through activation of NADPH oxidase and eNOS localized in membrane rafts and caveolae of bovine aortic endothelial cells. Am J Physiol Heart Circ Physiol 292: 954962, 2007.

43. Yang JP, Merin JP, Nakano T, Kato T, Kitade Y, and Okamoto $\mathrm{T}$. Inhibition of the DNA-binding activity of NF-kappa B by gold compounds in vitro. FEBS Lett 361: 89-96, 1995.

44. Yang X, Doser TA, Fang CX, Nunn JM, Janardhanan R, Zhu M, Sreejayan N, Quinn MT, and Ren J. Metallothionein prolongs survival and antagonizes senescenceassociated cardiomyocyte diastolic dysfunction: role of oxidative stress. FASEB J 20: 1024-1026, 2006.

45. Yao D, Gu Z, Nakamura T, Shi ZQ, Ma Y, Gaston B, Palmer LA, Rockenstein EM, Zhang Z, Masliah E, Uehara $\mathrm{T}$, and Lipton SA. Nitrosative stress linked to sporadic Parkinson's disease: S-nitrosylation of parkin regulates its E3 ubiquitin ligase activity. Proc Natl Acad Sci U S A 101: 10810-10814, 2004.

46. Ye B, Maret W, and Vallee BL. Zinc metallothionein imported into liver mitochondria modulates respiration. Proc Natl Acad Sci U S A 98: 2317-2322, 2001.

Address correspondence to: Prof. Litai Jin

School of Pharmaceutical Science

Wenzhou Medical University Wenzhou 325000

P.R. China

E-mail: jin_litai@126.com

Date of first submission to ARS Central, January 28, 2016; date of final revised submission, June 20, 2016; date of acceptance, July 13, 2016.

\begin{tabular}{rl|} 
& Abbreviations Used \\
2-OGD $=$ & 2-oxoglutarate dehydrogenase \\
2-OGDHC $=$ & 2-oxoglutarate dehydrogenase complex \\
$3-\mathrm{NT}=$ & 3-nitrotyrosine \\
$\mathrm{ANOVA}=$ & analysis of variance \\
$\mathrm{A} . \mathrm{U}$. & $=$ arbitrary units \\
$\mathrm{CVD}=$ & cardiovascular diseases \\
cyto-RoGFP $=$ cytosolic-localized redox-sensitive & GFP mutant \\
$\mathrm{DAPI}=$ & $4^{\prime}, 6$-diamidino-2-phenylindole \\
$\mathrm{DFO}=$ & desferrioxamine mesylate \\
\end{tabular}




\section{Abbreviations Used (Cont.)}

$\mathrm{DHE}=$ dihydroethidium

DMEM $=$ Dulbecco's modified Eagle's medium

DMHA $=$ dimethylhexylamine

$\mathrm{EF} \%=$ percent ejection fraction

ELISA $=$ enzyme-linked immunosorbent assay

EMSA $=$ electrophoretic mobility shift assay

$\mathrm{ESI}=$ electrospray ionization

$\mathrm{FS} \%=$ percent fractional shortening

GAPDH $=$ glyceraldehyde 3-phosphate dehydrogenase

$\mathrm{HW} / \mathrm{BW}=$ heart weight normalized to body weight

IL- $1 \beta=$ interleukin- $1 \beta$

IL-6 $=$ interleukin- 6

iNOS $=$ inducible nitric oxide synthase

LPS $=$ lipopolysaccharide

L-sh-p65 = LRV-U6-p65-CMV-EGFP

$\mathrm{LV}=$ left ventricle

LVEDD $=\mathrm{LV}$ end-diastolic dimensions

LVESD $=$ LV end-systolic dimensions

MMTS $=$ methyl methane thiosulfonate

MnTMPyP $=$ Mn(III) tetrakis 1-methyl 4-pyridyl porphyrin pentachloride

$$
\begin{aligned}
\mathrm{MPO} & =\text { myeloperoxidase } \\
\mathrm{MT} & =\text { metallothionein } \\
\text { MT-TG } & =\text { MT-transgenic } \\
\mathrm{NO} & =\text { nitric oxide }
\end{aligned}
$$

PAI-1 = plasminogen activator inhibitor-1

$\mathrm{PD}=$ Proteome Discoverer

$\mathrm{PRM}=$ parallel-reaction monitoring

$\mathrm{ROS}=$ reactive oxygen species

$\mathrm{SA}-\beta$-gal $=$ senescence-associated betagalactosidase

$\mathrm{SD}=$ standard deviation

sqRT-PCR $=$ semi-quantitative RT-PCR

$\mathrm{SRM}=$ selective reaction monitoring

TCA $=$ tricarboxylic acid

TGF- $\beta 1=$ transforming growth factor- $\beta 1$

TNF- $\alpha=$ tumor necrosis factor

TRADD $=$ TNFR1-associated death domain

$\mathrm{TU}=$ transducing units

TUNEL $=$ terminal deoxynucleotidyl transferase dUTP-mediated nick-end labeling

UHPLC $=$ ultra-high-performance liquid chromatography

$\mathrm{WT}=$ wild type 